\title{
Cognitive load increases anthropomorphism of humanoid robot. The automatic path of anthropomorphism.
}

\author{
Nicolas Spatola ${ }^{1}, \&$ Thierry Chaminade ${ }^{2}$ \\ ${ }^{1}$ Istituto Italiano di Tecnologia, Genoa, Italy. \\ ${ }^{2}$ Aix-Marseille Université, Institut de Neurosciences de la Timone, UMR 7289, CNRS, \\ Marseille, France.
}

Corresponding author:

Nicolas Spatola

Istituto Italiano di Tecnologia

Via Morego, 3016163 Genova

Italy

E-mail: Nicolas.spatola@iit.it 
Abstract. Humanoid robots are predicted to be increasingly present in the everyday life of millions of people worldwide. Humans make sense these artificial agents' actions mainly through the attribution of human characteristics, a process called anthropomorphism. However, despite a large number of studies, how the representation of artificial agents is constructed remains an open question. Here, we aim at integrating the process of anthropomorphism into the cognitive control theory, that postulates that we adapt resources management for information processing according to the current situation. In three experiments, we manipulated the cognitive load of participants while being observed by a humanoid robot to investigate how it could impact the online adaptation of the mental representation of the robot. The first two experiments indicated an online control of demanding resources in order to switch from an intentional to a physical representation, therefore inhibiting anthropomorphic, i.e. social, inferences. The third experiment investigated how the goals of the observing robot, i.e. "what" versus "why" is the robot observing, influences the effect of the cognitive load, showing that an explicit focus on its intentionality automatically biases cognitive processes towards anthropomorphism, yielding insights on how we mentally represent interacting robots when cognitive control theory and robots' anthropomorphism are considered together. 


\section{Introduction}

Interaction with humanoid robots (referred to simply as "robots" in this document ${ }^{1}$ ) is likely to become widespread, in space, time and settings, in the decades to come. Human-Robot Interaction (HRI) studies have demonstrated that humans tend to reason about robots through a process called anthropomorphism. Anthropomorphism is defined as the attribution of human characteristics to non-human entities such as animals (Eddy et al., 1993; Wynne, 2006) or robots (Nyangoma et al., 2017; Złotowski et al., 2015). Robots' anthropomorphism is not an anecdotal process as it entails not only how humans represent robots (Darling, 2017; Eyssel and Kuchenbrandt, 2012; Kuchenbrandt et al., 2013; Spatola et al., 2019), but also how robots impact humans' cognition and behavior (Riether et al., 2012; Spatola et al., 2019, 2018). In this paper, we investigate how cognitive control mechanisms influence robots' anthropomorphism. In particular, cognitive load has been shown to modulate how people explain others' actions and make inferences about them in terms of physical or mental properties (Rosset, 2008). For example, people tend to attribute more divine intentions to natural events and produce more teleological interpretations when they experience a shortage of cognitive resources (Kelemen and Rosset, 2009). We further develop the dichotomy between physical and mental social cognition systems and the modulating role of cognitive resources.

\subsection{Physical and social processing}

Humans interpret changes in the world as either physical (e.g., the dynamics of moving objects according to Newtonian laws) or mental (e.g., the understanding of voluntary actions by intentional agents) processes. Philosopher Daniel Dennett (Dennett, 2009) proposed that individuals adopt a specific stance depending on the phenomenon being observed. Whenever they try to predict physical phenomena such as the trajectory of a ball being kicked, they take

\footnotetext{
${ }^{1}$ The term "robot" has different acceptations, some including industrial robotic devices producing repetitive manipulations, but the focus of the present study is on human-like robots.
} 
a physical stance, relying on empirical rules. However, more complex phenomena such as human behaviour cannot be efficiently described using these physical rules. The intentional stance describes another mode of representation that relies on mentalizing, and in particular the attribution of mental states such as intentions. Intentional and physical representations are mutually exclusive, so that when the intentional system is active the physical system is inactive (Jack et al., 2013a). This dichotomy of cognitive systems for making sense of moving entities is reminiscent of the proposal of two core cognitive processes (Darlow and Sloman, 2010; Evans, 2008; Evans and Stanovich, 2013). According to this model, cognition may be dichotomized as Type 1 "automatic" and Type 2 "controlled" processes. These two cognitive systems differ in terms of resource demands, that influence the speed of processing, the amount of information being processed, etc... Type 1 requires a lower activation of working memory through the use of stored representations and is more rigid than Type 2, making it a fast processing process. Type 2 requires cognitive decoupling from Type 1 and a higher amount of cognitive resources, resulting in a slower but controlled processing of information. Interconnections, and sometimes overlap, between the brain social areas and the default mode network, for example the posterior superior temporal sulcus and medial prefrontal and posterior cingulate cortices, or the insula and amygdala (Goodson, 2005; Hansen et al., 1976; Mars et al., 2012), have led some authors to posit that humans' default cognitive state could rely on social inferences (Iacoboni et al., 2004; Jack et al., 2013b; Saris et al., 2020; Tavares et al., 2008). The reason would be that social functioning is necessary for human survival (Eisenberger and Cole, 2012; Humphrey, 1976; Valtorta et al., 2018), and therefore has been subjected to positive evolutionary selection. In contrast, explicit cognitive control mechanisms, including inductive and causal forms of reasoning characteristics of the physical system, are more cognitively demanding and slower than implicit processes, and are limited by the capacity of working memory (Evans, 2008). 
Robots' anthropomorphism could reproduce this dichotomy, in the sense that the observer could accumulate information based on the intentional (Type 1) or physical (Type 2) cognitive system. According to Epley and colleagues, representing a non-human agent's actions using human's intentional representation system (anthropocentric schemas) would be an easier and faster strategy to explain, understand and react to its behaviour (Epley et al., 2007). This view was supported by later studies (Meyer et al., 2012; Spunt and Lieberman, 2013), and is in line with the Computer As Social Actor theory (Nass and Moon, 2000), that states that people apply rules and social expectations to computers mindlessly, except when these agents violate their expectations (Epley et al., 2008, 2007; Marsh et al., 2014). However, these attributions don't necessarily imply that people believe that computers have intentions or free will. Such phenomenon would be more likely to occur when the context triggers an individual to use stored representations that don't require conscious awareness (Langer, 1992). With humanoid robots, the distinction between mindless and intentional attributions is more complex than with computers or other non-anthropomorphic tools on which the CASA theory applies (Coeckelbergh, 2012; Gunkel, 2019, 2018; Levy, 2009; Neely, 2014). This is due to their anthropomorphic embodiment, their interactivity or their "social" purpose, which is more likely to trigger intentional interpretation of their behaviour.

From a cognitive point of view, anthropomorphism could thus be conceptualized as a process in which the internal representation of the incoming sensory information about an acting agent, that include contradictory information such as a human-like shape made of mechanical elements in the case of humanoid robots, are continuously generated according to the social (type 1) or physical (type 2) cognitive systems. However, as the representation is, hypothetically, construed online with the observation, the process has to be continuous, or, to put it differently, iterative. Indeed, each neuronal or computational continuous process is iterative depending on the time or biological scale in which it is investigated. This iterative characteristic supposes two 
axioms. First, if the process is iterative, the default anthropomorphic reasoning (relying on intentional representations, Type 1) can be surpassed by non-anthropomorphic reasonning (physical cognition system, Type 2), finally resulting in a conflict between the two representational systems while it is still cognitively malleable. Second, if anthropomorphism is the default reasoning pathway, it has to be actively counteracted, which requires additional cognitive resources. Therefore, the capacity to select Type 2 type of reasoning to process incoming information about the changes observed in the surroundings should be proportional to the amount of resources available for cognitive control.

\subsection{The role of cognitive control in anthropomorphism}

As for many other dual-processing accounts of reasoning, judgment, and social cognition (Evans, 2008), we propose that anthropomorphism should be considered within the cognitive control framework. Cognitive control entails the processing of the relevant information given a specific context (Engle, 2002). It is an active system pertaining to the ability to select or inhibit perceptual or mental elements. Unlike automatic processes which take place automatically, cognitive control, by definition, controlled and, as a consequence, is slower and requires more resources (Ridderinkhof et al., 2004a, 2004b). Given certain contexts, the cognitive control system will inhibit automatic processing (Augustinova et al., 2018; Sharma et al., 2010). However, cognitive resources, that are required for this inhibition, are limited. The cognitive load theory formalizes how the system allocate the limited amount of cognitive resources. It describes the storage and processing of information in working memory and the integration of new information (Leppink, van Gog, Paas, \& Sweller, 2015; Paas, van Merriënboer, \& Adam, 1994). For example, more resources are available when individuals perform a single task compared to two tasks simultaneously (Moscovitch, 1994), or in the presence of competition between interfering cues (Augustinova et al., 2018; MacLeod, 1992; Stroop, 1935). In tasks in which individuals have to inhibit an automatic process to select a valid answer, such as the 
Stroop task, the cognitive control is required to inhibit the competing cue, and we propose that this inhibition process could apply to anthropomorphism (Urquiza-Haas and Kotrschal, 2015).

Some authors hypothesized that the tendency to anthropomorphize artificial agents should increase in a situation of high cognitive load (Waytz et al., 2010), but this has never been demonstrated. Urquiza-Haas and Kotrschal proposed that a high cognitive load would interfere with the suppression of the default social cognition system when the latter is triggered by perceptual information (Urquiza-Haas and Kotrschal, 2015). If we consider that anthropomorphism is the default system, i.e. that the intentional system is an automatic process while the physical system is at rest, the perception of robots should rely, at first, on social cognition processes (Type 1) favouring anthropomorphic inferences. However, because robots are not human, cognitive control should inhibit this automatic path, that is inappropriate for mechanical artefacts, and activate the physical system (Type 2) (Darlow and Sloman, 2010; Evans and Stanovich, 2013). Reliance on this physical system would in turn inhibit anthropomorphic inferences resulting in robots being correctly considered as objects. In the proposed framework, this inhibition requires cognitive resources and, as such, depends on the amount of cognitive resources available during the observation of the artificial agent's actions (figure 1).

As we mentioned earlier, the building of mental representation is conceptualized as an iterative process generated by cerebral, cortical as well as subcortical, computations (Cunningham and Zelazo, 2007). When interacting with a robot, one has sensory information about its shape, colour, size, design, movements, etc... Eyes closed, one can build a mental, coherent and stable, representation of the robot, that includes characteristics that may or may not be accurate (Broadbent, 1984; Dumontheil et al., 2016; Heine et al., 2006). This process is central for robots' anthropomorphism as it is this coherent and stable mental representation, and not the robot itself, that is used because people reason about the robot to answer, for example, 
questions like "Do you like this robot?" or "Do you find this robot warm?” (Lee et al., 2005). In the framework we propose here, cognitive control should modulate which elements of this mental representation arise from the intentional system and from the physical system when building this coherent and stable representation. In the case of high cognitive load, leading to a lack of resources for cognitive control, more time would be required to inhibit the default intentional system, resulting in more social elements being included in the representation and, as a consequence, increased anthropomorphism.

\subsection{The purpose of observation affects anthropomorphism}

Caporael (Caporael, 1986; Hansen et al., 1976) argued that when observing and forming a mental representation of an agent, the purpose of this observation has to be taken into account. For instance, Spunt and Lieberman (2013) showed that attributing mental properties to an agent was modulated by cognitive load only when participants were prompted to attributed a motive to observed action ("Try to understand why the agent is doing this action") but not when the observer adopts a more descriptive perspective ("Try to understand how the action is doing this action"). Similarly, Chaminade et al (ref) found that neural correlates of action observation in the mirror system are more activated when the robot's actions have to be interpreted in mental terms ("Rate the emotion of the robot") versus mechanistic terms ("Rate the motion of the robot"). In other words, individuals tend to anthropomorphize more when they are explicitly asked to activate the intentional system (Meyer et al., 2012; Spunt and Lieberman, 2013). However, contrary to predictions arising from the current proposal, Spunt and Lieberman (2013) demonstrated that the attribution of mental properties to a target decreased with cognitive load when individuals were instructed to focus on the intention of the observed agent. This inadequacy between our hypothesis and Spunt and Lieberman results may be disentangle by a study by de Lange and colleagues (de Lange et al., 2008) that showed that asking participants to reflect deliberately on goals and intention could bias how mirror system and 
mentalizing areas are functionally connected (de Lange et al., 2008) thus impacting information integration from these two systems (Sperduti et al., 2014; Van Overwalle and Baetens, 2009). In other words, explicitly reflecting on the agent's goal would lead to override the automatic intentional process of mentalization (Spunt et al., 2015), a process likely to require active cognitive control. We therefore propose that priming observers to reflect on the purpose of the agent's action will produce different results depending on the cognitive load, such that high cognitive load would degrade intentional processes when participants are explicitly requested to reason about the intention of a target agent (Lin et al., 2010; Spunt and Lieberman, 2013). Still, in the absence of specific instructions one would tend to attribute more intentions to agents under high cognitive load (Kelemen and Rosset, 2009). As proposed by Keysers and Gazzola (Keysers and Gazzola, 2007) inferring others' mental states "is often neither purely intuitive nor purely reflective, which is why knowledge of both mechanisms should be combined" (Keysers and Gazzola, 2007, p.194). But when individuals are explicitly required to reflect on the purpose of an agent, the automatic processing is inhibited in favour of a controlled, and conscious, process (Moors and De Houwer, 2006; Wegner and Bargh, 1998). In other words, the intention identification task, when explicitly instructed, would require a large amount of cognitive resources, for example because the conflict between intentional and physical processes then requires to be explicitly addressed to achieve the task, and would therefore be impaired by a lack of cognitive resources.

\subsection{Present study}

We propose a model, inspired by descriptions of a competition between fast and automatic versus slow and controlled cognitive processing, to describe processes underlying robot's anthropomorphism. When building of a mental representation of a robot, the fast process is attribution of human-like intentions to make sense of the robot's action (intentional stance) while the slow process relies on interpreting the movements in mechanical terms 
(physical stance). Importantly, the model isn't static but dynamic, that can be understood as a loop in which the representation of the agent is built through iterations of competitions and integrations of the two processes (Figure 1). For each iteration, individual characteristics forming the mental representation of the agent could result either on the social or physical processes, considered as mutually exclusive (Schilbach et al., 2008). Alternatively, both processes could be activated in parallel information treatment pipelines that weight automatic and controlled processes to provide a common output: the parallel-competitive processing model (Smith and DeCoster, 2000). Our current hypothesis, focused on the cognitive load and the goal of observation, would apply in both framework and we do not aim at investigating this epistemological question in the present study.

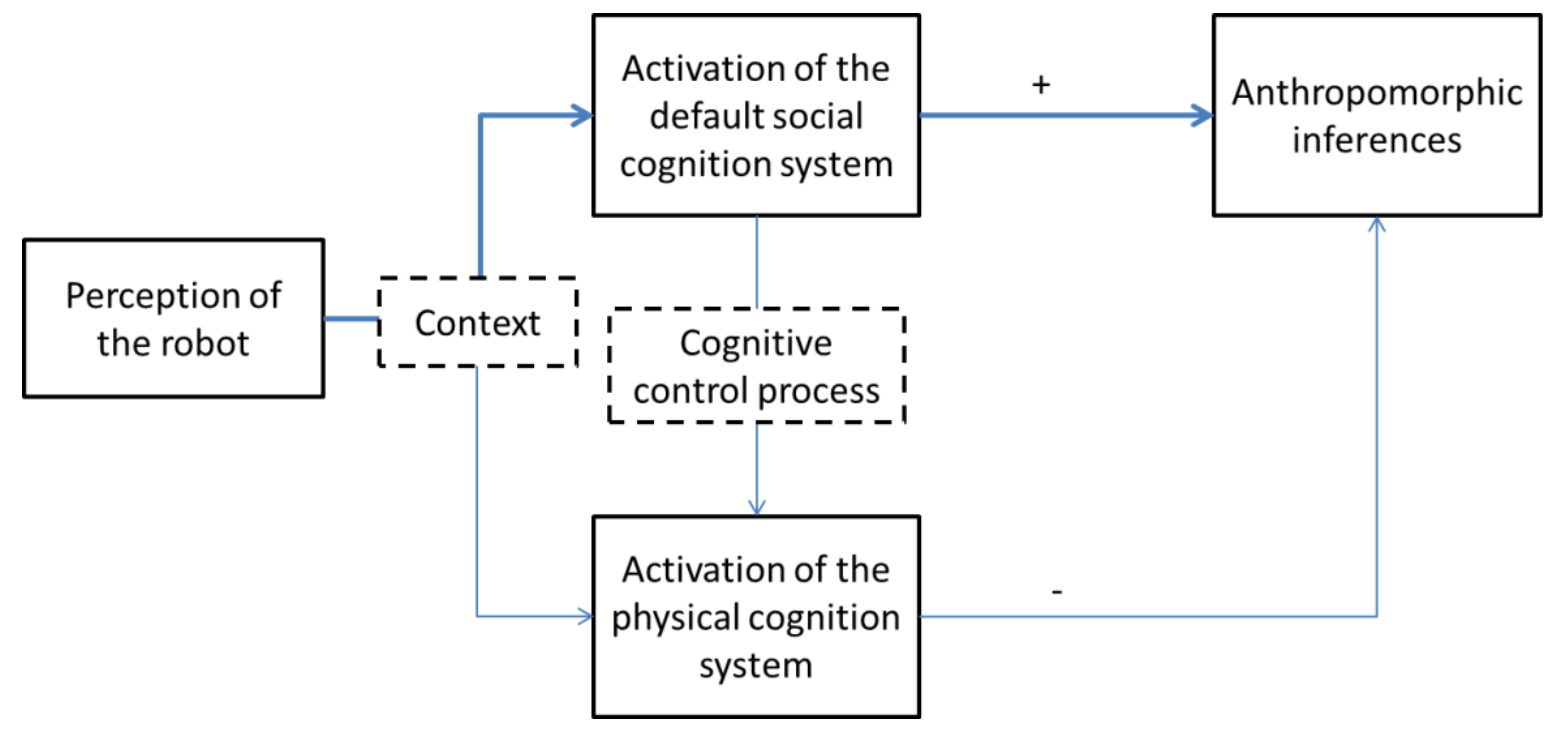

Figure 1. Illustration of our model for robot anthropomorphism

The studies performed to test this model primarily focus on the effect of cognitive control on anthropomorphism, investigated by modulating the availability of cognitive resources. In experiments 1 and 2, we manipulated the level of cognitive load while participants watched a video presenting a robot and subsequently measured anthropomorphic inferences. Participants were either in a low or a high cognitive load condition. We hypothesized that in situations of 
high cognitive load, participants would not be able to inhibit the default intentional process and would anthropomorphize the robot being presented to a larger extent than in low cognitive load situations. Also, higher the anthropomorphism would be associated with higher cognitive load felt by participants. In the third experiment, we test the hypothesis that this process is influenced by the purported purpose of the robot's observation (Caporael, 1986; Hansen et al., 1976; Meyer et al., 2012; Spunt and Lieberman, 2013), manipulated by instructions the direct participants attention to intentional or physical properties of the robot. We hypothesize that when participants are explicitly required to explain the behaviour of the agent in intentional terms, anthropomorphism should increase. We further test the complete model in the form of a decision tree that explains the anthropomorphism as the function of the cognitive load and the goal of evaluation.

\section{Experiment 1}

\subsection{Method}

Participants were 83 males, 42 females and 4 others $\left(M_{\text {age }}=20.6, S D=4.8\right)$ recruited online. The experiment was processed with Qualtrics. Participants took part in this experiment on a voluntary basis. We estimated the requested sample size with $G^{*}$ Power, with a medium effect size, a $0.05 \alpha$, power set at 0.90 and a $1 / 1$ between-group ratio (Faul et al., 2007). The requested sample size was 108.

The experimenter informed participants that the experiment was a memory test. Instructions provided the organization of the experiment: 1) a questionnaire; 2) a sequence of numbers to memorize; 3) a video to watch about which questions would be asked subsequently. 
First, they had to complete the Negative Attitudes towards Robots Scale (NARS) (Syrdal et al., 2009). This scale was used to assess that both experimental groups were comparable considering attitudes toward robots.

Second, they were randomly assigned either to the high cognitive load $(n=66)$ or the low cognitive load ( $n=63)$ group. In both group they were informed that a sequence of numbers would appear for 30 seconds, their task being to memorize it (Gilbert and Hixon, 1991; Sherman et al., 1998; Sherman and Frost, 2000; Wehling et al., 2008). In the high cognitive load experimental condition, the sequence was composed of 9 digits randomly selected (i.e. “214087539”, “257142809”, “952176034”). In the low cognitive load experimental condition, the sequence was composed of 4 digits also randomly selected (i.e. " $2148 ”$, " $2571 "$, "9521”).

Third, a screen instructed participants that they would see the video and had to keep the digits in memory. The video presented a NAO robot interacting with a human, an object and another NAO for 1.36 minutes. The video came from an Aldebaran Nao presentation video (from https://www.youtube.com/watch?v=rSKRgasUEko). We choose the video format to intentional processes as it has been shown that perceiving a moving stimulus with animacy characteristics increases the social cognition network activation (Wheatley et al., 2007), as supported by seminal studies on anthropomorphism (Berry et al., 1992; Heider and Simmel, 1944). For instance, Berry and colleagues (1992), using the well-known Heider and Simmel (1944) animated film, demonstrated that disruption of shapes' animation reduced anthropomorphism while strobe-like disruption of patterns eliminated it. The authors argued that smooth dynamic information was used as evidence of intentionality. Also, the NAO robot displays facial features such as eyes and mouth that strongly reinforce its human likeliness (DiSalvo et al., 2002). To avoid any priming effect, the video was cut so as to not display any logo and sound. After watching the video participants had to write the sequence of digits and judge their agreement about two proposals to evaluate the (subjective) cognitive effort induced by the experimental 
condition on a likert scale(i.e. "Regarding the memory task you performed, the task was very complex", "Regarding the memory task you performed, you provided a very high mental effort to solve it”, $\alpha=.85$ ) (Brünken et al., 2004; Leppink et al., 2015; Paas et al., 1994).

Finally, they completed a questionnaire adapted from Haslam dehumanization taxonomy (Haslam, 2006; Spatola et al., 2019, 2018) and the Robotic Social Attribute Scale (RoSAS) (Carpinella et al., 2017). We used these questionnaires as a measure of anthropomorphism.

Attitudes toward robots. The NARS scale is designed to explain participants' evaluations of robot in a Human-Robot Interaction (HRI) study. The NARS scale is made of 3 subscales: 1) Relational attitudes $(\alpha=.73)$ (e.g. "I would feel very nervous just standing in front of a robot"), 2) Future/social influence $(\alpha=.70)$ (e.g. "I am concerned that robots would be a bad influence on children"), 3) Actual interactions and situations $(\alpha=.75)$ (e.g." I would feel relaxed talking with robots"). For each dimension, participants rated whether they agreed or disagreed (from 1 to 7 ).

Anthropomorphism measure 1. Participants also filled out the humanness scale based on Haslam's dehumanization taxonomy with human uniqueness (e.g., moral sensibility) and human nature (e.g. interpersonal warmth) as the humanization dimensions $(\alpha=.80)$, and the animalistic dehumanization (e.g., irrationality) and mechanistic dehumanization (e.g., inertness) as the dehumanization dimensions $(\alpha=.68)$. Again, for each dimension, participants rated whether they agreed or disagreed (from 1 to 7 ) to characteristics attributed to the robot presented on the video.

Anthropomorphism measure 2. Finally, participants filled out the RoSAS with warmth (e.g., happy) ( $\alpha=.80)$, competence (e.g. capable) $(\alpha=.70)$, and discomfort (e.g., scary) ( $\alpha=$ .83) dimensions. For each dimension, participants rated whether they agreed or disagreed (from 1 to 7$)$ to characteristics attributed to the robot. 


\subsection{Results}

\subsubsection{Control analyses}

On participants' attitudes of participants toward robots investigated with NARS prior to the actual experiment, a multivariate ANOVA showed that there was no difference between participants assigned to high $v s$. low cognitive load experimental conditions on any of the three dimensions: Relational attitudes, $F(1,128)=.12, p=.735, \eta_{p}^{2}<.01,95 \% C I[-.29, .42]$, Future/social influence, $F(1,128)=1.23, p=.270, \eta^{2} p=.01,95 \% C I[-.58, .16]$, and Actual interactions and situations, $F(1,128)=.26, p=.613, \eta^{2} p<.01,95 \% C I[-.34, .57]$.

As expected from experimental manipulation, participants assigned to the high load cognitive load condition (compared to low cognitive load condition) declared higher cognitive effort during the task, $F(1,128)=37.11, p<.001, \eta^{2}=.23,95 \% C I[1.16,2.28]$, than low cognitive load participants.

\subsubsection{Cognitive load effect on anthropomorphic attributions}

To test the effect of the cognitive load manipulation (high vs. low) on humanness scores (humanization, de-humanization) and RoSAS scores (warmth, competence and discomfort), we conducted a multivariate ANOVA including the cognitive effort measure as a covariate. Results showed no significant results, all $p \mathrm{~s}>.05$.

To test whether the cognitive load reported by participants was related to NAO anthropomorphic and human traits attributions, we conducted regression analyses with the level of cognitive effort as predictor. Results showed that the higher the declared load, the higher the warmth, $b=.29, t(128)=3.41, p=.001, \mathrm{R}^{2}=.08,95 \% C I[.08, .30]$, competence, $b=.276 p=$ $.007, \mathrm{R}^{2}=.06,95 \% C I[.04, .23]$, and human traits attributions, $b=.34, t(128)=4.06, p<.001$, $\mathrm{R}^{2}=.12,95 \% C I[.10, .29]$, and the lower the discomfort, $b=-.22, t(128)=-2.59, p=.011, \mathrm{R}^{2}$ 
$=.05,95 \% C I[-.27,-.04]$ and dehumanization attributions, $b=-.30, t(128)=-3.50, p=.001$, $\mathrm{R}^{2}=.09,95 \% C I[-.24,-.07]$.

\subsection{Discussion experiment 1}

The present study investigated the hypothesis that, in situations of high cognitive load, the limitation of resources required to inhibit the default intentional process would lead participants to anthropomorphize the robot being presented on the video to a larger extent than in low cognitive load situations,. Results showed no evidence for an effect of the controlled level of cognitive load. However, we found a positive relation of the subjective cognitive load on anthropomorphic and human traits attributions. The presents result supports an influence of subjective cognitive load on robots anthropomorphism. However, in the absence of significant effect from the objective manipulation of the cognitive load, this interpretation has to be taken with caution. An alternative explanation for this absence of effect could be that the cognitive load experimental manipulation was not sufficient to produce an effect. The second experiment tested whether this result could be confirmed by an active interfering task.

\section{Experiment 2}

We replicated the previous study using an active interfering task maximizing the cognitive load effect. We replaced the memory task by a mental calculation task presented during the video (Ayres, 2001; Lamberts et al., 2000). The main difference between the memory task and the mental calculation task is the presentation of new stimuli during the video which increases the cognitive load when the robot representation is being built. As previously, we hypothesized that in a situation of high cognitive load, participants wouldn't be able to inhibit the default intentional process and would thus anthropomorphize the robot displayed on the video to a larger extent than participants in a low cognitive load situation. Also, the higher the 
level of cognitive effort declared by participants, the higher the anthropomorphic attributions would be observed.

\subsection{Method}

Participants were 75 males, 31 females and 2 others (Mage $=19.2, \mathrm{SD}=2.5$ ) recruited online. The experiment was processed with Qualtrics in groups. Participants took part in this experiment on a voluntary basis. We estimated the requested sample size with G*Power, with a medium effect size, a $0.05 \alpha$, power set at 0.90 and a $1 / 1$ between-group ratio (Faul et al., 2007). The requested sample size was 108 .

The experimenter informed participants that they will take part in a cognitive test. Instructions provided the organization of the experiment: 1) a questionnaire (NARS scale), 2) a video (i.e. the same video as in experiment 1) to watch on which, subsequently, 3) questions would be asked. In contrast to the first experiment, one-digit numbers (e.g., 5) were displayed during the video. Participants were randomly assigned to one of our two conditions. Half the participants were instructed to sum all numbers (i.e. low cognitive load experimental condition), while the other half the instruction was to multiply them (i.e. high cognitive load experimental condition). We choose these two arithmetic tasks because they both rely on the same memory network (Geary et al., 1986), however multiplication requires more cognitive resources than addition (Sweller, 1994). The value, location and timing of digits were identical across conditions.

After the video participants had to write the results of their calculation and rate the same two proposals as in experiment 1 to evaluate the (subjective) cognitive effort induced by the experimental condition (i.e. "Regarding the memory task you performed, the task was very complex", "Regarding the memory task you performed, you provided a very high mental effort 
to solve it”, $\alpha=$.88) (Brünken et al., 2004; Leppink et al., 2015; Paas et al., 1994). Finally, they completed the same questionnaires as experiment 1.

Attitudes toward Robots. Again, we used the NARS scale made of 3 subscales: 1) Relational attitudes $(\alpha=.71), 2)$ Future/social influence $(\alpha=.69), 3)$ Actual interactions and situations $(\alpha=.67)$.

Anthropomorphism measure 1. After watching the video, participants filled out the humanness scale based on Haslam's dehumanization taxonomy with humanization $(\alpha=.76)$, and dehumanization $(\alpha=.70)$ dimension.

Anthropomorphism measure 2. Finally, participants filled out the RoSAS with warmth ( $\alpha=.77)$, competence $(\alpha=.50$, non-reliable), and discomfort $(\alpha=.78)$ dimensions.

\subsection{Results}

\subsubsection{Control analyses}

A multivariate ANOVA showed no significant prior differences between participants in high cognitive load $v s$. low cognitive load experimental conditions on the three dimensions of the NARS filled prior to video exposure: Relational attitudes, $F(1,107)=.01, p=.927, \eta^{2} p$ $.01,95 \% C I[-.38, .42]$, Future/social influence, $F(1,107)=.64, p=.424, \eta^{2} p=.01,95 \% C I[-$ $.22, .51]$, and Actual interactions and situations, $F(1,107)=.38, p=.539, \eta^{2} p<.01,95 \% C I[-$ $.33, .62]$. It should be mentioned that the Cronbach's alpha reliability estimate was questionable for the last two dimensions (Brown, 2002; Cortina, 1993; Cronbach, 1951). As expected, results showed that participants in the high load cognitive load condition (compared to low cognitive load condition) declared higher cognitive effort during the task, $F(1,107)=95.60, p<.001, \eta^{2} p$ $=.47,95 \% C I[2.47,3.72]$. 


\subsubsection{Cognitive load effect on anthropomorphic attributions}

To test the effect of the cognitive load manipulation (high vs. low) on humanness scores (humanization, de-humanization) and RoSAS scores (warmth, competence and discomfort), we conducted a multivariate ANOVA. Results showed that participants in the high cognitive load condition attributed more human traits, $F(1,107)=48.91, p<.001, \eta^{2} p=.32,95 \% C I[.69,1.24]$, more warmth, $F(1,107)=40.58, p<.001, \eta^{2} p=.28,95 \% C I[.82,1.55]$, and more competence, $F(1,107)=7.73, p=.006, \eta^{2} p=.07,95 \% C I[.12, .73]$, to the robot compared to participants in the low cognitive load condition. All the other $p \mathrm{~s}>.05$.

To test whether the level of cognitive effort declared by participants could explain the effect of the experimental condition on anthropomorphic attributions we conducted mediation analyses using PROCESS plugin in SPSS (Bolin, 2014; Fritz et al., 2012; Hayes and Rockwood, 2017; Hayes and Scharkow, 2013; Preacher and Hayes, 2004; Shrout and Bolger, 2002; Snyder, 1961; Yzerbyt et al., 2018) for on humanness and warmth scores, with experimental conditions (high vs. low cognitive load) as a predictive factor (X) and the level of declared cognitive effort as mediating factor $(\mathrm{M}$, bootstrap $=10000)($ MacKinnon et al., 2004) (Figure 2).

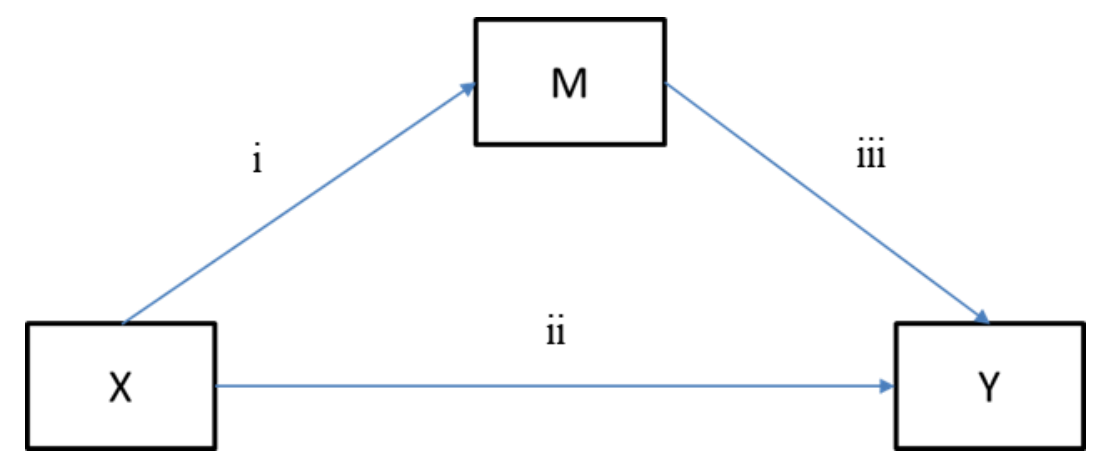

Figure 2. Mediation model

Humanization. In the mediation model the direct path between experimental conditions (X) and human traits attributions (corresponding to (ii) in Figure 2) remained significant, $b=.44, t(106)=2.36, p=.020,95 \% C I[.07, .84]$ (Figure 3). Results also showed that the higher 
the declared level of cognitive effort (M), the more participants attributed humanness traits to the robot presented in the video $(\mathrm{Y}), b=.44, t(106)=4.76, \mathrm{p}<.001,95 \% C I[.12, .29]$ (corresponding to (iii) in Figure 2). The mediation path $\mathrm{X} \rightarrow \mathrm{M} \rightarrow \mathrm{Y}$ was also significant $b=$ $.42,95 \% C I[.23, .65]$. In other words, similar to experiment 1 , participants in the high cognitive load condition attributed higher human traits to the robots compared to participants in the low cognitive load condition and this effect was explained, at least in part, by the higher level of cognitive efforts reported by participants.

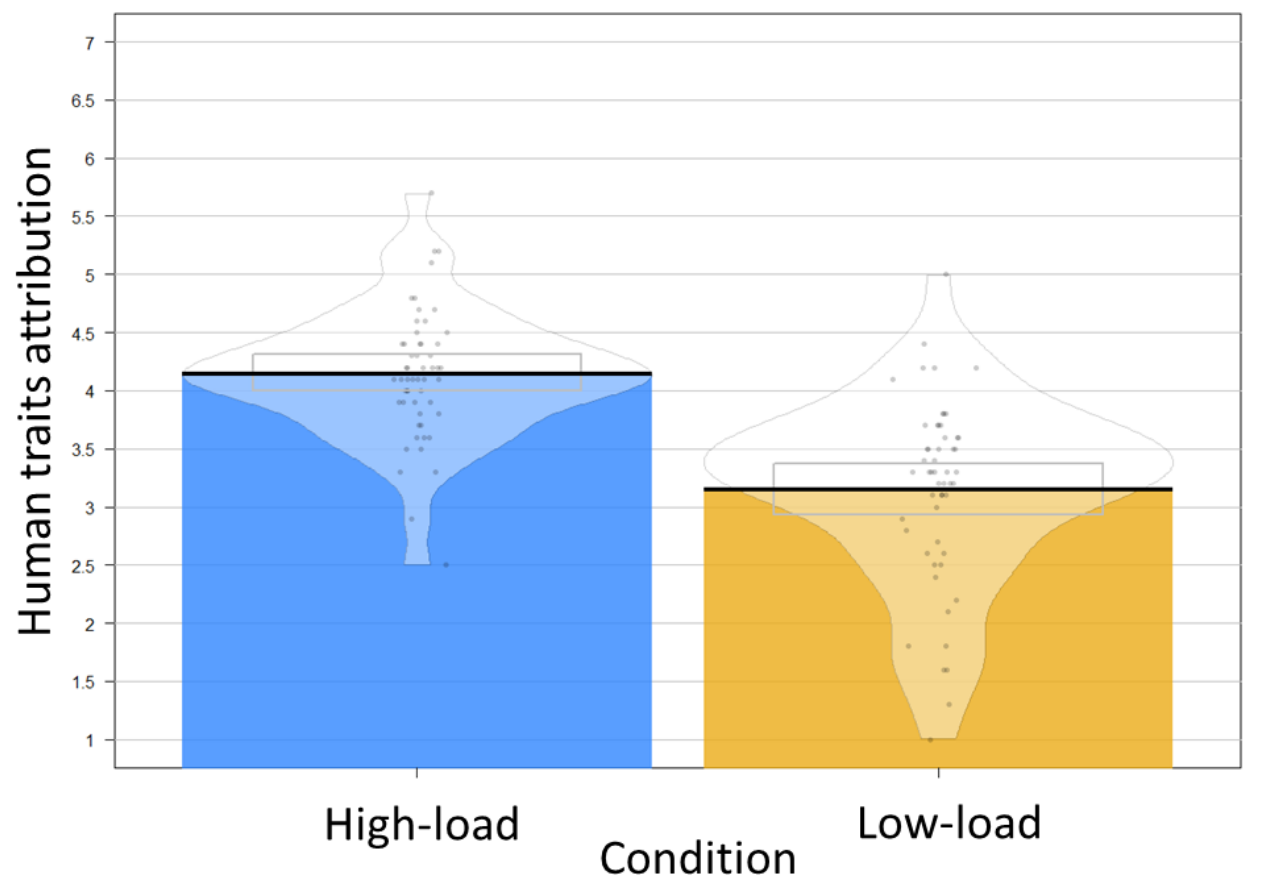

Figure 3. Human traits attribution as a function of the cognitive load (high vs. low).

Warmth. In the mediation model including the warmth scores as dependant variable, the effect of the experimental condition became non-significant, $b=.06, t(106)=.21, p=.833$, $95 \% C I[-.55, .69]$. We found that the higher the level of subjective cognitive effort $(\mathrm{M})$, the more participants attributed warmth characteristics $(\mathrm{Y})$ to the robot presented in the video, $b=$ $.45, t(106)=3.22, p=.002,95 \% C I[.09, .36]$. Our analyses argue for a mediation of the effect 
of the experimental condition on warmth attribution by the declared level of cognitive effort, $b$ $=.70,95 \% C I[.28,1.15]$.

Competence. The following results should be taken with caution as the Cronbach alpha was not reliable for this dimension. In the mediation model, the effect of the experimental condition on competence attributions became non-significant, $b=-.51, t(106)=-1.74, p=.084$, $95 \% C I[-.82, .05]$. Also, we found that the higher the declared level of cognitive effort (M), the more participants attributed competence characteristics (Y) to the robot presented in the video, $b=.52, t(106)=3.53, p<.001,95 \% C I[.08, .28]$. Our analyses showed a significant mediation of the effect of the experimental condition on competence attribution by the declared level of cognitive effort, $b=.61,95 \% C I[.30 ; .93]$.

\subsubsection{Exploratory analyses}

We conducted an ANOVA including the cognitive load experimental condition (high vs. low) and the experiment (experiment 1 vs. experiment 2) as independent variables and the declared cognitive effort as dependant variable to test whether the change of interfering task increased the cognitive effort declared by participants. We found an interaction between experiment and cognitive load condition, $F(1,236)=9.21, p=.003, \eta^{2} p=.04$. Post-hoc contrasts with Bonferroni correction showed that while there was no difference between studies in the low cognitive load conditions, $F(1,233)=2.01, p=.157, \eta^{2} p=.01,95 \% C I[-.16,1.01]$, participants in experiment 2 high load condition declared higher cognitive effort compared to their counterparts in experiment $1, F(1,233)=33.03, p<.001, \eta^{2} p=.12,95 \% C I[1.11,2.72]$.

\subsection{Discussion experiment 2}

The second study aimed at replicating and strengthening the results of first study investigating whether, in situations of high cognitive load, participants would fail to inhibit the default intentional process and therefore anthropomorphize the robot being presented to a larger 
extent than in low cognitive load situations. In accordance with this hypothesis, we found significant differences in the evaluation of the robot according to both the objective and subjective levels of cognitive load. In addition, the increase of positive anthropomorphic inferences (warmth, competence) and human traits attribution (humanization) was significantly mediated by the reported level of cognitive effort (all confidence intervals excluded 0 ).

In line with our hypothesis, results of experiment support an important influence of available cognitive resources when making an anthropomorphic judgement about a humanoid robot. As fewer resources become available, positive scales increase and negative scales lower values. These results argue for an automatic intentional processing reflected in higher anthropomorphism when fewer control resources are available. And corollary, the reduced positive anthropomorphism observed in the low cognitive load condition reflect, within the present dual-route framework, increased reliance on controlled cognition, namely, the adoption of a physical stance to make judgements about the robot being shown on the video.

Using a more active task than mere memory maintenance, the second study supports a role of the amount of available cognitive resources in the establishment of mental representation of robots and, as a consequence, of the robot's anthropomorphism that result from this representation.

\section{Experiment 3}

Experiment 1 and 2 aimed at investigating the effect of cognitive control on robot's anthropomorphism by modulating available cognitive resources. Taken together the results are in line with the hypothesis that people manage to inhibit the anthropomorphic inferences only when sufficient cognitive resources are available. Cognitive control process allows a switch from the automatic intentional to the physical cognition network resulting in less anthropomorphic attributions. However, as mentioned in the introduction, some authors argued 
that the purported goal of the robot was of prime importance when explaining the behaviour of the target entity in anthropomorphic terms (Caporael, 1986; Hansen et al., 1976; Meyer et al., 2012; Spunt and Lieberman, 2013). In experiment 3, we aimed at disentangling how focusing participants' attention on the intention (Spunt and Lieberman, 2013) or purpose (Gazzola et al., 2007) of the robot's actions may result in different influence of cognitive load, while still considering the intentional stance as the default processing mode (Dennett, 1971; Spunt et al., 2015). We asked participants to watch a video presenting a robot with either the objective of answering the question "Why the robot is doing that?" (condition focusing on the goal of the robot's action in intentional terms) or the question "What is the robot doing" (condition focusing on the goal of the robot's action in physical terms). With this experimental manipulation, we assumed that deliberate reflection on intentions would increase anthropomorphism (de Lange et al., 2008). We also introduced a control condition of passive observation (observation goal condition) in which participants were informed that they will have to watch a video with no further information. The purpose was to avoid participants to focus on the robot's actions. As previously we manipulated the cognitive load of participants (high vs. low cognitive load conditions).

In line with our previous results, participants in the high cognitive load condition would anthropomorphize more the robot than participants in the low load condition. We also hypothesized that participants in the "why" goal condition would anthropomorphize the robot more than the "what" condition as the focus would be made on intentional causal attribution in the former and on a more pragmatic level of analysis in the latter (Dennett, 1971; Heil and Heil, 2019; Meyer et al., 2012; Spunt and Lieberman, 2013). In addition, both should result in higher anthropomorphic attributions compared to the (passive) observation goal condition because the video would be treated as an interfering element in the task resulting in a lowered level of attention. However, an impairment of anthropomorphic attribution should occur under high 
cognitive load and when participants are primed by the "why" goal as reflecting deliberately on intention would impair mentalization process, in line with Spunt and Lieberman (2013). Similarly, participants would attribute more anthropomorphic traits to the robot in the "what" goal condition in the high cognitive load condition compared to the low load condition) as they would not focus on intentions. Therefore, we hypothesized that the goal should significantly influence the cognitive load effect.

\subsection{Method}

Participants were 93 males, 206 females and 53 others or who preferred not to answer (Mage $=22.2, \mathrm{SD}=8.7)$ recruited online. The experiment was processed with Qualtrics. Participants took part in this experiment on a voluntary basis. We estimated the requested sample size with $G^{*}$ Power, with a medium effect size, a $0.05 \alpha$, power set at 0.90 and a $1 / 1$ between-group ratio (Faul et al., 2007). The requested sample size was 350 .

First, building upon Spunt and Lieberman (Spunt and Lieberman, 2013), the experimenter informed participants that the experiment consisted in a memory test. Instructions provided the schedule of the experiment: a video to watch on which, subsequently, questions would be asked. Participants were then randomly assigned to one of the two experimental conditions. Half of the participants were instructed to remember an easy numbers sequence (e.g., 555-5555; further mentioned as the low load condition) while the other half had to remember a difficult numbers sequence (e.g., 813-5647; further mentioned as the high load condition).

Second, we manipulated the goal factor by instructing participants to have specific goals while watching the video: i) actively recognize the action ("You will have to describe WHAT the robot is doing"; the what goal condition), ii) to understand the motive behind the action ("You will have to describe WHY the robot is doing these actions"; the why goal condition), or iii) to 
passively observe the video (the observation goal condition). The same video as in experiment 1 and 2 was used to ensure the comparison between the experiments.

Third, after the video participants had to recall the numbers sequence they had to memorized and to evaluate the robot on the Human-Robot Interaction Evaluation Scale (HRIES) (Spatola et al., 2020) which contains four dimensions of robot evaluation including Sociability (4 items, e.g., Warm, $\alpha=.90$ ), Agency (4 items, e.g., Self-reliant, $\alpha=.73$ ), Animacy (4 items, e.g., Alive, $\alpha=.63$ ), and Disturbing ( 4 items, e.g., Creepy, $\alpha=.83$ ). This scale makes it possible to evaluate static, in motion or interactive robots on a broad spectrum of anthropomorphic attributions. For each item, participants rated whether they agreed or disagreed (on a scale from 1 to 7 ) on characteristics to the iCub robot (presented on a picture above the scale), i.e. "For each trait, you will have to evaluate whether, according to you, it corresponds or not to the robot that is presented to you". For each trait, a 7-points slider scale was presented from 1 "not at all" to 7 "totally". We chose the HRIES in the third experiment because the scale has been developed as an aggregated and updated version of the two anthropomorphic measures used previously during the current project. It also makes it possible to test the same hypothesis with a slightly different but overall similar measure.

\subsection{Results}

We conducted a MANOVA including the type of goal ("what" vs "why" vs observation) and the cognitive load condition (high load vs low load) as independent variables and the HRIES dimensions scores as dependent variables. Results showed a main effect of the goal on Agency $F(2,349)=55.11, p<.001, \eta^{2} p=.21$, Sociability $F(2,349)=34.49, p<.001, \eta^{2} p=.08$, and Animacy $F(2,349)=7.22, p=.001, \eta^{2} p=.04$ dimensions, but not on the Disturbing dimension $F(2,349)=1.81, p=.165, \eta^{2} p=.01$. Pairwise contrasts with Bonferroni correction showed that all comparisons were significant except for the "what" and "why" goal conditions on Animacy dimension (see table 1). Participants attributed anthropomorphic characteristics to a higher 
extent in the "what" and "why" goal conditions compared to the observation one and in the "why" goal condition compared to the "what" one.

\begin{tabular}{cclcccc} 
& \multicolumn{2}{c}{ Goal } & t statistics & p.value & \multicolumn{2}{c}{$95 \%$ CI } \\
\hline \multirow{3}{*}{ Disturbing } & Observation & What & $F(1,232)=.008$ & .999 & -.41 & .39 \\
& Observation & Why & $F(1,231)=2.38$ & .332 & -.13 & .67 \\
& What & Why & $F(1,235)=3.09$ & .276 & -.39 & .41 \\
\hline \multirow{3}{*}{ Agency } & Observation & What & $F(1,232)=40.19$ & $<.001$ & -1.27 & -.58 \\
& Observation & Why & $F(1,231)=68.50$ & $<.001$ & -1.69 & -1.00 \\
& What & Why & $F(1,235)=7.71$ & .010 & -0.76 & -.08 \\
\hline \multirow{3}{*}{ Sociability } & Observation & What & $F(1,232)=8.91$ & .008 & -1.07 & -.12 \\
& Observation & Why & $F(1,231)=27.63$ & $<.001$ & -1.56 & -.61 \\
& What & Why & $F(1,235)=5.91$ & .036 & -.965 & -.02 \\
\hline \multirow{3}{*}{ Animacy } & Observation & What & $F(1,232)=6.08$ & .033 & -.77 & -.02 \\
& Observation & Why & $F(1,231)=13.08$ & .001 & -.95 & -.20 \\
& What & Why & $F(1,235)=1.32$ & .715 & -.55 & .19 \\
\hline
\end{tabular}

Table 1. Contrasts' statistics with Bonferroni correction for each anthropomorphic dimension.

We also found significant interactions between the goal and the cognitive load on Agency, $F(2,349)=23.12, p<.001, \eta^{2} p=.12$, Sociability $F(2,349)=8.34, p<.001, \eta^{2} p=.05$, and Animacy $F(2,349)=6.79, p=.001, \eta^{2} p=.04$. To further investigate these interactions, we used a tree-based classification model to predict the values of the dependent variables (Disturbing, Agency, Sociability, Animacy) with a Chi-squared Automatic Interaction Detection (CHAID) algorithm. This algorithm identifies the independent variables (predictors) that have the strongest interaction with the dependent variable. Non-significant predictors are merged in one category. This process allows extracting a hierarchy of predictors from the data taking into account different levels of interaction between the goal and cognitive load. Overall results showed a primacy of the goal (observation vs. "why" vs. "what") in all anthropomorphic dimensions except disturbing (see figure $5,7,8$ ). The effect of the cognitive load was therefore conditioned by the type of goal, in support of our hypothesis. 
Agency. Results on Agency attributions showed that the three goals (Observation, "what", "why") primed the effect of the cognitive load on attributions (Figure 4).

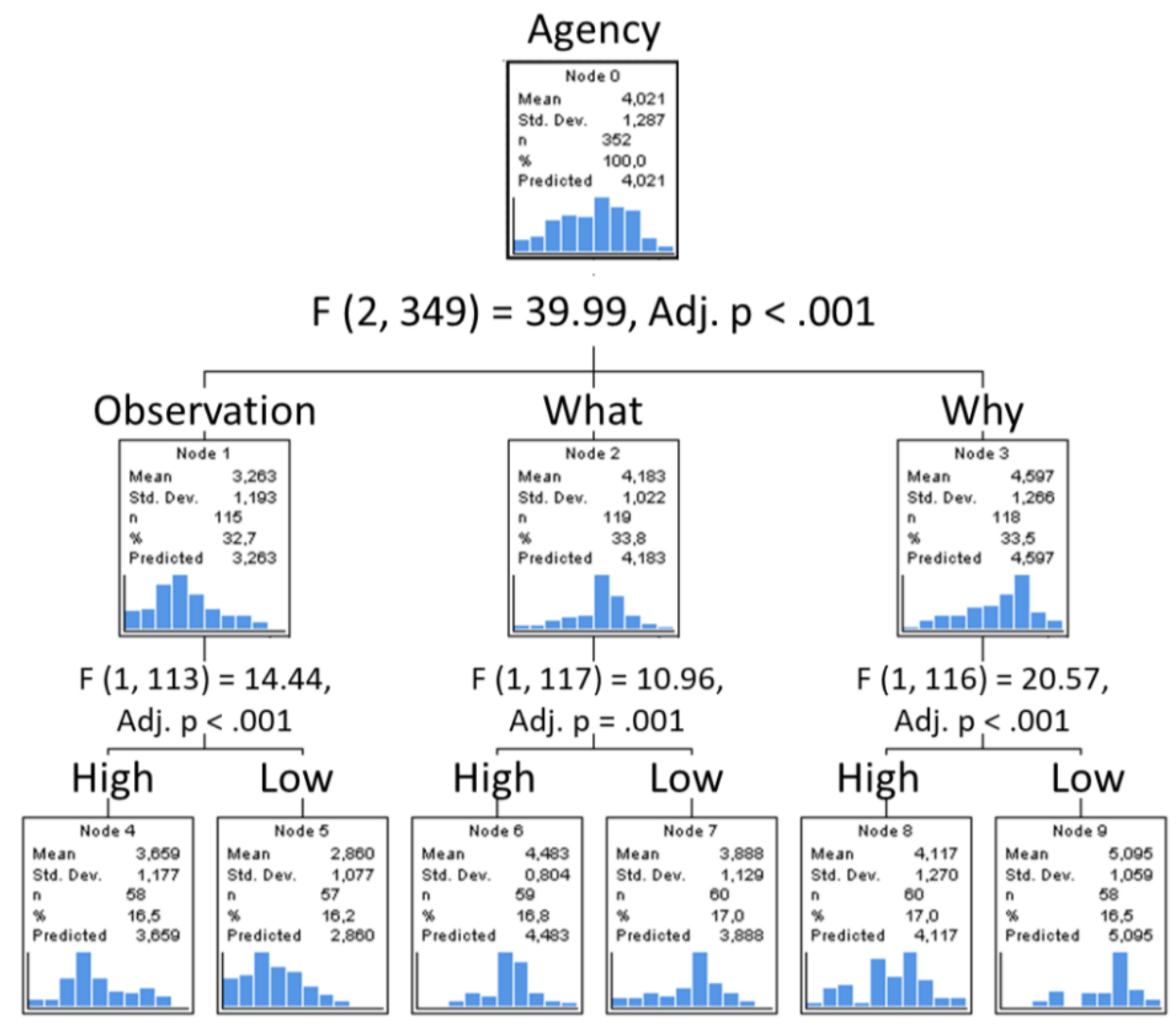

Figure 4. Decision tree to predict agency traits attribution as the function of goal and cognitive load

While participants attributed more traits to the robot in the observation $F(1,113)=14.44, p<$ $.001, \eta^{2} p=.11,95 \% C I[.48,1.22]$ and what $F(1,117)=10.96, p=.001, \eta^{2} p=.09,95 \% C I[.24$, .95] goals conditions in the high compared to low cognitive load; in the why goal condition participants attributed less agency traits in high (compared to low) cognitive load condition, $F(1,116)=20.57, p<.001, \eta^{2}=.15,95 \% C I[-1.41,-.55]($ Figure 5). 


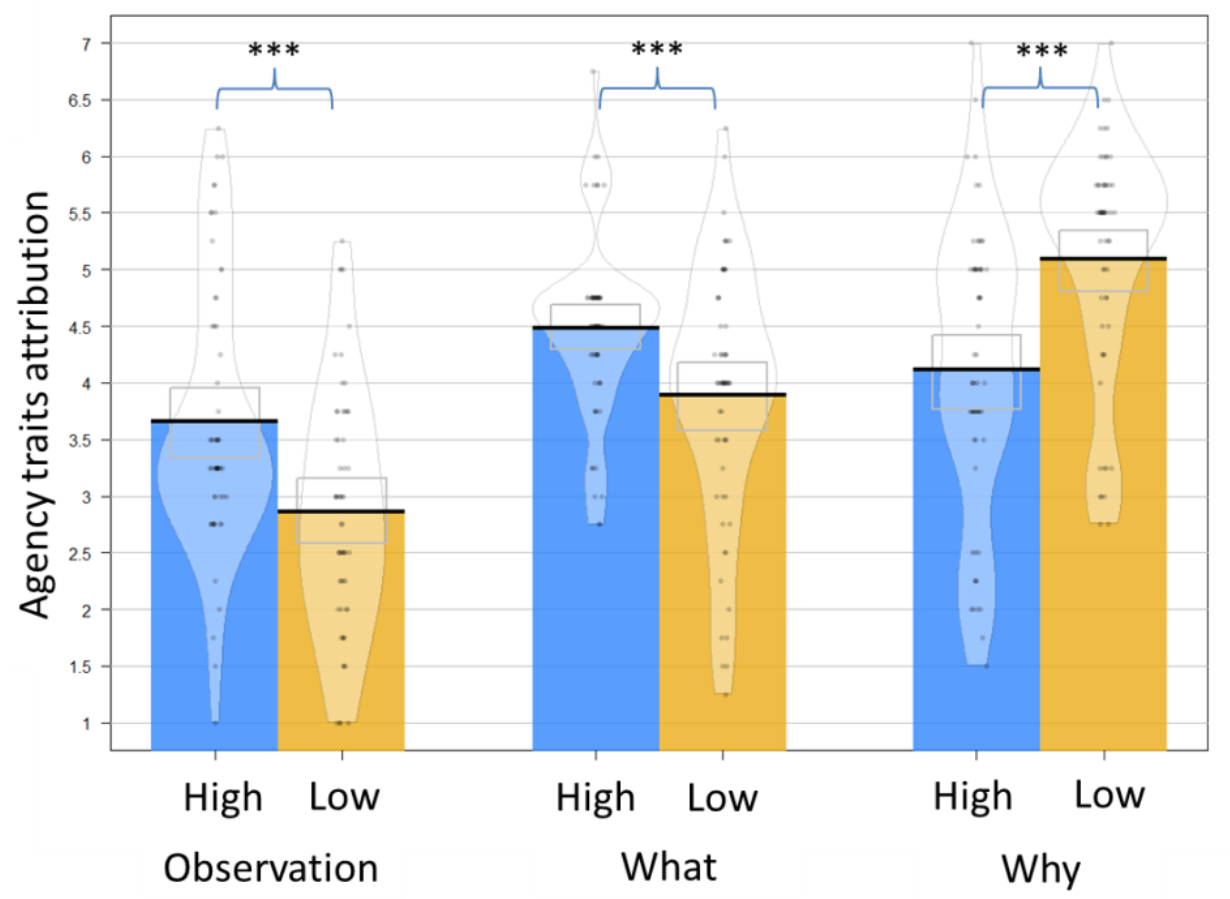

Figure 5. Agency traits attribution as the function of the goal (observation vs "what" vs "why") and cognitive load (high vs low). *** $: \mathrm{p}<.001$.

Sociability. On the sociability dimensions, the goal primed the effect of the cognitive load in the observation and "why" but not "what" goals (Figure 6). While there was no effect of cognitive load in the "what" goal condition $F(1,117)=2.68, p=.104, \eta^{2} p=.02,95 \% C I[-.09$, .98], participants in the observation condition attributed more sociability traits to the robot in the high (compared to low) cognitive load condition $F(1,113)=9.79, p=.002, \eta^{2} p=.08,95 \% C I$ $[.31,1.40]$, and the reverse pattern was true for the " $w h y$ " goal condition $F(1,116)=5.81, p=$ $.017, \eta_{p}^{2}=.05,95 \% C I[-1.27,-.13]$. 


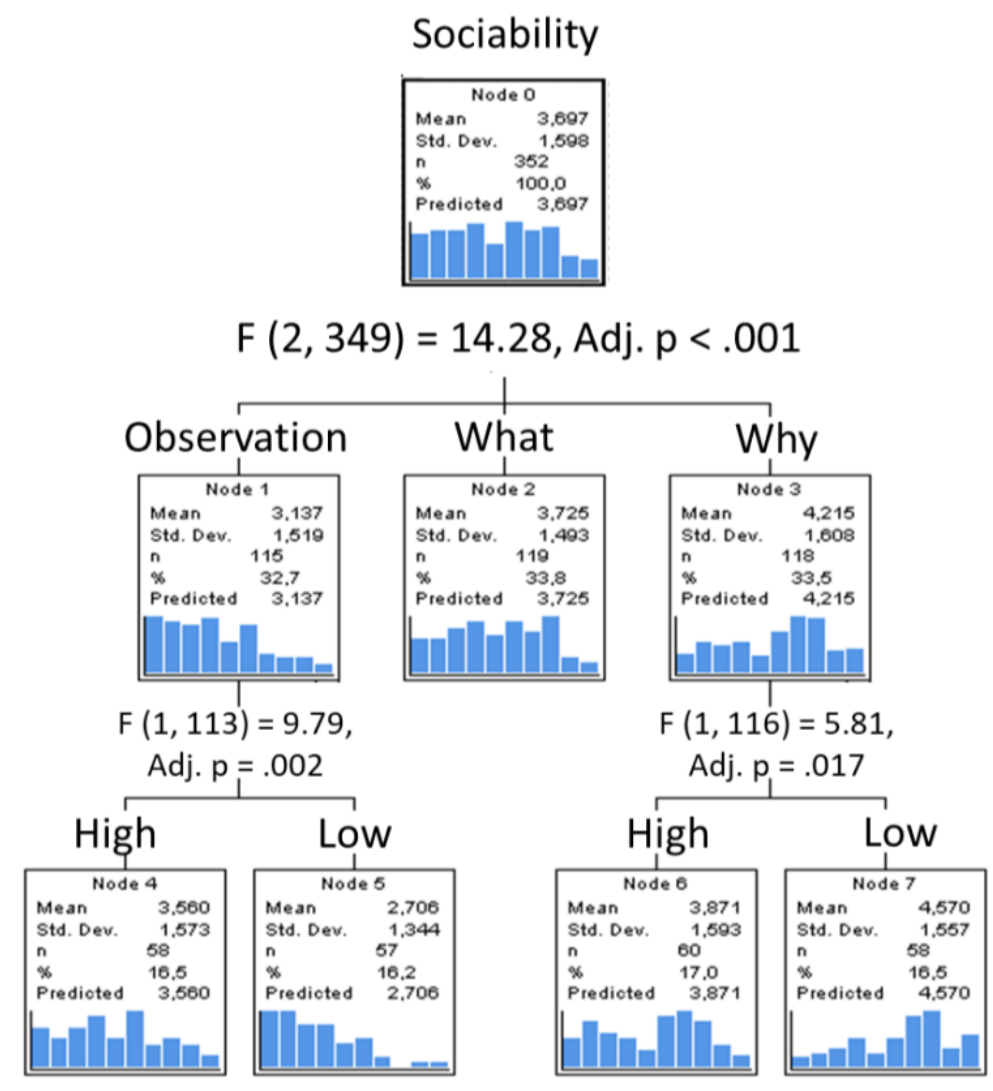

Figure 6. Decision tree to predict sociability traits attribution as the function of goal and cognitive load

Animacy $^{2}$. Finally, results on Animacy attributions showed that the effect of the load was only dependent of the observation goal while there was no effect in the "what" and "why" conditions $\left(p_{\mathrm{s}}>.05\right)$ (Figure 7). Attribution increased in high (compared to low) load in the observation condition, $F(1,113)=6.08, p=.015, \eta^{2} p=.05,95 \% C I[.12, .99]$.

${ }^{2}$ The Cronbach alpha of the Animacy dimension was not reliable (Cronbach, 1951), therefore results on this dimension should be taken with caution. 

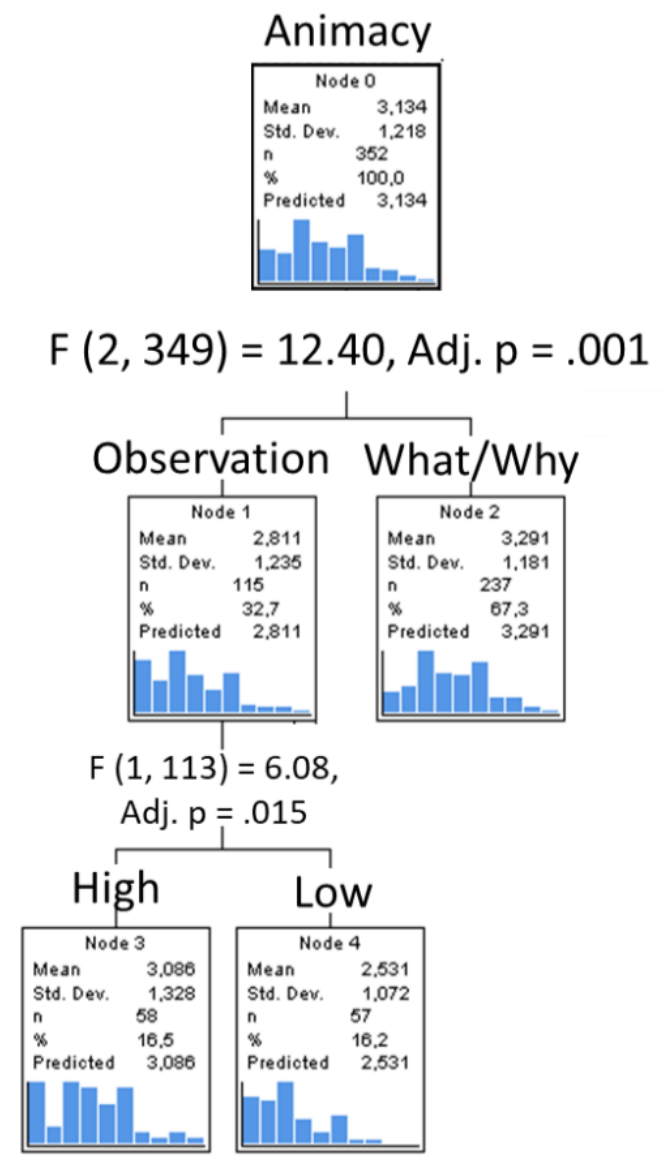

Figure 7. Decision tree to predict animacy traits attribution as the function of goal and cognitive load

\subsection{Discussion Experiment 3}

Experiment 3 aimed at evaluating how the type of interpretative goal would modulate the influence of cognitive load on anthropomorphic attributions. Besides, we aimed at investigating whether the goal was the modulator or was modulated by the cognitive load.

Firstly, in line with previous studies (Meyer et al., 2012; Spunt and Lieberman, 2013),we found that individuals attributed more anthropomorphic traits when they were explicitly asked to ("why" goal condition) compared to the condition priming an emphasis on the action ("what" goal condition). In addition, both resulted in higher attribution anthropomorphic traits than the mere observation goal condition. 
Secondly, we found a significant interaction between goal and cognitive load. Participants in the observation and "what" goals attributed more anthropomorphic characteristics to the robot in high compared to low load condition while it was the opposite in the "why" condition. This last result, in line with Spunt and Lieberman findings, indicates that when people are primed to reason about the intention of an agent, the increase of cognitive load impairs anthropomorphism (Spunt and Lieberman, 2013). However, when the attention is focused on another characteristic of the agent behaviour such as "what the agent is doing" as well as during mere observation, high cognitive load results in higher anthropomorphic attributions compared to low cognitive load (similar to the results in experiment 1 and 2).

When people deliberately reflect on intention, the interaction between mirror neurons and mentalizing systems, which play a central role in social cognition, is changed (de Lange et al., 2008; Sperduti et al., 2014; Van Overwalle, 2009; Van Overwalle and Baetens, 2009). In the "why" goal condition, the automatic synergy between mentalization and motor resonance could be bypassed and become a controlled process. This interpretation is in line with fMRI research demonstrating increased activity in mentalization areas and a loss of the synergy between the mirror and mentalizing systems when participants are explicitly instructed to understand the intention of an agent (Chaminade et al., 2012; de Lange et al., 2008; Spunt and Lieberman, 2013). Spunt and colleagues, in a further study, showed that mentalization is the default mode of human brain, and would be highly sensitive to the type of task demand (Spunt et al., 2015). Therefore, if the attribution of intention becomes a controlled process requiring resources, cognitive resources depletion could impair the automatic process of intention attribution (Engström et al., 2017) or, as named by Dennett, the adoption of the intentional stance (Dennett, 1971). 


\section{General discussion}

Across 3 experiments, we investigated the process of anthropomorphism in relation to the cognitive control theory. More specifically, we grounded our hypotheses in a model that postulates two cognition systems in human perception. First, the intentional system is an automatic process based on the social perception of the environment and the activation of social representation (social schema, social scripts). Second, the physical system is a controlled process focusing on physical properties of the stimuli and relying on empirical rules (Jack et al., 2013a; Mars et al., 2012; Tavares et al., 2008). With respect to this framework, we proposed that human perception when perceiving human-like artificial agents', such as humanoid robots, behaviours, would be first intentional resulting in anthropomorphic attributions but could be actively controlled to switch to physical reasoning (Heider and Simmel, 1950; Jack et al., 2013a; Tavares et al., 2008) and representations more specific of the artificial target. Finally, we aimed at investigating the interaction between cognitive load and goal of evaluation, especially when individuals are required to focus on purported intentional motivations of the observed artificial agent. In line with previous studies (de Lange et al., 2008; Spunt and Lieberman, 2013), we hypothesized that when individuals observe a robotic agent, a mental representation of this artificial agent is built based primarily on processes from the social cognition system that could be actively bypassed by the physical cognition system if sufficient cognitive resources are available. (Experiments 1, 2). However, this social vs. physical inferences could be modulated by the goal of observation as explicitly focusing on mental states countervails the standard modus operandi of switching from an automatic to a controlled process (Experiment 3).

In line with previous research on mental representation (Huang and Awh, 2018), our results (experiment 1 and 2) demonstrate that judgments about robots are based on a pre-structured mental representation rather than a reactivation and reevaluation of the percept (i.e. the physical 
content of the video). This view of anthropomorphism is in accordance with Epley and colleagues' model which posits that the core of anthropomorphism is the representation of the object rather than the object itself (Epley et al., 2007; Waytz et al., 2010). However, the building of the representation should be considered as a dynamic process. Indeed, if the process was only one shot, we would not observe a switch from a representation mainly based on social information (anthropomorphic) to a representation mainly based on physical information (nonanthropomorphic). In other word, the representation of the agent remains malleable as long as the observation goes. Therefore, we could assume that the longer the observation, the more structured and the less malleable the representation (Carlston and Smith, 2007). The reason is that the accumulation of information runs against the malleability of the representation. The higher the amount of information, the higher the inertia with which the weight of an information is pondered by the social $v s$. physical representations.

Mental representation of robots are often biased by the pop-culture and unrealistic positive or negative expectation given the limited access to direct interaction and information about these new artificial agents. This phenomenon has been described as the "Hollywood robot syndrome" (Sundar et al., 2016) and could explain the actual high level of inter-individual differences in attitudes (e.g. interest, fear) and willingness to interact with robots. Also, because our results argue for the importance of the mental representation, even inaccurate, methods to assess these mental representations and their malleability are crucial. Here we used explicit measures but we could expect that implicit measures, in particular relying on participants' physiological measure (Chaminade, 2017), could be better suited. Explicit measures operate at the conscious level and generally use self-reports such as questionnaires, while implicit measures rely on unconscious and automatic processes, for example using reaction time paradigms or implicit association tests (De Houwer et al., 2009). Research suggests that implicit measures might constitute better predictors of future intentions and behaviours (Kurdi et al., 
2019), and thus be more representative of real representations than explicit declarations. Implicit measures have also proved to be well suited to predict the behavioural consequences of individuals' implicit representations either with humans (Friese et al., 2008; Kurdi et al., 2019) or robots (Spatola and Wudarczyk, 2020, 2021).

As postulated by the cognitive control framework, in which we anchored our hypotheses, the amount of cognitive resources available has an important influence on the process of anthropomorphism (Lavie, 2010). As hypothesized, we found that cognitive load might modulate the amount of anthropomorphic attribution to the robot. The lower the amount of cognitive resources available, the higher the anthropomorphic attributions. These results are in line with decades of social psychology literature, for example pertaining to stereotypes, demonstrating that the lack of cognitive resources favours the reliance on automatic processes to reason about the environment (Sherman et al., 1998). Taken together, results from studies 1 and 2 support the model that postulates anthropomorphism relies on intentional processes as the default mechanism that can only be controlled when sufficient resources are available. In experiment 2 in particular, results show that the higher the amount of cognitive resources taken by the interfering task, the higher the anthropomorphic attributions.

Anthropomorphism makes it easier to analyse robot interactions because it is based on the best known model of human observers: the human (Epley et al., 2007), even though the perceptual input is degraded comparing to observing a real human acting (Chaminade et al., 2005). To illustrate the process, we may consider the case of peripheral vision as a metaphor for the mediating effect of the cognitive load on anthropomorphism. While observers do not have "a clear image" of peripheral objects represented in their visual system, they subjectively perceive these peripheral objects as clear because they can complete their representation with similar elements stored in memory, even though the actual sensory information is not complete or accurate (O'Rourke, 1993). Completing a representation of a robot by using accessible 
elements that can easily be retrieved from memory, in this precise case human representations, can be seen as a computational strategy to complete and stabilize the representation. This would be even truer when the observer lacks cognitive resources, further "blurring the image". However, when individuals have sufficient cognitive resources to deeply reason about the robot ("to clean the image"), individuals would refer to a more accurate and thus less anthropomorphic representation of the robot, relying mainly upon what Dennett called "the physical/design stance". The result would be a switch from intentional to physical processes caused by the rationalization of the robot as an artefact. Saving resources using human schemas (i.e., social cognition) would therefore no longer be necessary and detailed analysis (i.e., physical cognition) would become possible.

Also, results of experiment demonstrated that the goal of the observers can modulate how the cognitive load biases anthropomorphic attributions. Priming participants to adopt, on purpose, the intentional stance resulted in a reversed pattern with lower anthropomorphism in high (compared to low) cognitive load situation. This suggests to further consider how the experimental control of a default process could bias this process (Spunt et al., 2015). It is in accordance with the CASA hypothesis (Nass and Moon, 2000). While individuals tend to use anthropomorphic attribution to think about a non-human (e.g. robotic) agent, asking them explicitly whether they actually consider them as anthropomorphic will result in a denial of such attribution. However, how this denial occurs remained poorly understood. Whether it could arise from reactance (Brehm, 1981; Steindl et al., 2015), ironic rebound (Wegner and Schneider, 2003) or contrast comparison between humans and robots (Brown and Hewstone, 2005; Spatola and Urbanska, 2019) remained unanswered.

Finally, using different cognitive load manipulations in the 3 experiments, we may now assume that the effect does not depend on a specific task but more generally on a common process within the cognitive control framework, putatively in relation to the cognitive load. 
These results, again, argue for the crucial role of the context in anthropomorphism. In different contexts, different representations and, as a consequence, judgments, of the same robot can be produced by the same individual. Taking into account this information we could define strategies to purposefully enhance or reduce robots' anthropomorphism. For instance, it could be considered as positive to anthropomorphize a host robot in a store. Therefore, programming the robot to transmit an important amount of information could reduce the availability of cognitive resources for customers and increase the anthropomorphic attributions. This can be done by mixing the cues that the social cognition system will treat automatically such as facial or body gestures providing emotions or orientation indices, the logorrhoea and the type of semantic (e.g. pragmatic vs emotional). In this example, the increase of the number of stimuli is twofold: i) the increase of the social signals, triggering the activation of the social cognition system, and ii) the increase of the amount of signals to process, requiring more cognitive resources alleviating the counter-anthropomorphic process. Going further, because anthropomorphism is sensitive to the context, we could posit that the representation could be modulated by the type of cognitive load sources, for example, positive or negative semantic related stimuli (Hermans et al., 2003; Iida et al., 2012; Meisner, 2012) and as such modulate the experience with the robot as a social agent but also the evaluation of the actions of the robot.

\section{Limits}

Some limits have to be discussed. First, we cannot consider here the gap in the attribution of anthropomorphic characteristics between a robot and a human performing the same actions. Thus, even if robots are more anthropomorphic in high cognitive load condition, the distance between them and a complete anthropomorphism (i.e., comparison to the human prototype) can't be evaluated.

Second, our study doesn't make it possible to study the effect of repeated interactions and the hypothesis of a reduction in anthropomorphic attributions over time. In light of recent brain 
imaging studies, it could, therefore, be assumed that the difference on structures such as the parietal temporal junction (associated to the attribution of intentionality) or the hypothalamus subnuclei (associated to the social bonding) highlighted by Rauchbauer and colleagues (Rauchbauer et al., 2019) in the HHI and HRI comparisons only appear at certain moments of the interaction, for example, if no differences of activity were found at the beginning of the interaction (Spatola et al., n.d.).

Third, our studies only emphasize the "interpretative" aspect of anthropomorphism. However, Fisher identified two different ways to engage in anthropomorphic thinking: 1) interpretative, the attribution of anthropomorphic characteristics based on observation and 2) imaginative, as the representation of fictional entities detached from concrete perception. Therefore, we could assume that the cognitive cost or motivation to switch from the social to the physical cognition network could be influenced by this prior. The design of the artificial agent also can be a huge source of variability (Duffy, 2003). It could be argued that attributions could be modulated by the anthropomorphic characteristics of the design such as the presence of a mouth or eyes (DiSalvo et al., 2002). Still, understanding the actions of robots, even nonanthropomorphic ones, takes advantage of the intricate brain mechanisms that developed to understand the actions of our conspecifics, that is the mirror neuron and mentalization systems (Gazzola et al., 2007).

Fourth, while participants were on the usual of average age for psychological study, this could limit the generalizability of the results. The reason is that age is an important factor in people's relation to technology. Indeed, acceptance or knowledge are two dimensions correlated to age. Considering that in our proposed model, knowledge about robots is an important part of the use of an anthropomorphic representation (or not) (Epley et al., 2008, 2007; Waytz et al., 2010), age could be a moderator interesting to take into account. 
Fifth, we agree that other factors not investigated in present study, such as the human-like appearance (Nyangoma et al., 2017; Salem et al., 2013) influencing motor resonance processes (Chaminade et al., 2012, 2010), the social context (Spatola et al., 2019, 2018), or the observer's knowledge about robots (Epley et al., 2008, 2007; Waytz et al., 2010), could reinforce or interfere with the weighting of social $v s$. physical processes. In other words, the more the context provides social information or triggers social processing, the more it is difficult for the cognitive control to inhibit the default social cognition path.

Finally, as we mentioned in introduction section, the nature of the relations between social and physical processes remains an open question. In the parallel-competitive model, both processes are activated in parallel information treatment pipelines that weight automatic and controlled information to provide a single output (Smith and DeCoster, 2000). In the default system theories, the social cognition system produces the initial output that can, or not, be corrected at a second stage by the physical cognition system, similar to evidence accumulator models that are computed until the production of the final output (Evans and Stanovich, 2013). The present results do not make it possible to disentangle the two proposals.

\section{Conclusion}

Anthropomorphism is fundamental to human-robot (and other non-human agents) interactions as it helps to make sense of the artificial agent's behaviour and is activated by default (as a dominant response) and need to be actively inhibited. To embed anthropomorphism in cognitive control theory and the dual-route (social $v s$. physical cognition) framework makes it possible to predict better the situation in which one would engage in anthropomorphism. In addition, the observation goal needs to be taken into account, as focusing on intention modifies how people adopt an intentional stance toward non-human agents. Forcing a voluntary control over a process automatically activated by default, in our case the 
attribution of intentionality, tends to inhibit the automatic route as cognitive resources are reduced by the conscious control.

\section{Compliance with Ethical Standards}

The study was conducted in accordance with the Code of Ethics of the World Medical Association (Declaration of Helsinki).

\section{Conflict of Interest:}

The authors declare that they have no conflict of interest.

\section{Open Practices}

All data are publicly available via the Open Science Framework and can be accessed at https://osf.io/6zq8n/

\section{References}

Augustinova, M., Silvert, L., Spatola, N., Ferrand, L., 2018. Further investigation of distinct components of Stroop interference and of their reduction by short response-stimulus intervals. Acta Psychol. (Amst). 189, 54-62. https://doi.org/10.1016/j.actpsy.2017.03.009

Ayres, P.L., 2001. Systematic Mathematical Errors and Cognitive Load. Contemp. Educ. Psychol. 26, 227-248. https://doi.org/10.1006/ceps.2000.1051

Berry, D.S., Misovich, S.J., Kean, K.J., Baron, R.M., 1992. Effects of Disruption of Structure and Motion on Perceptions of Social Causality. Personal. Soc. Psychol. Bull. 18, 237-244. https://doi.org/10.1177/0146167292182016

Bolin, J.H., 2014. Hayes, Andrew F. (2013). Introduction to Mediation, Moderation, and 
Conditional Process Analysis: A Regression-Based Approach. New York, NY: The Guilford Press. J. Educ. Meas. 51, 335-337. https://doi.org/10.1111/jedm.12050

Brehm, S.S., 1981. Psychological reactance and the attractiveness of unobtainable objects: Sex differences in children's responses to an elimination of freedom. Sex Roles 7, 937-949. https://doi.org/10.1007/BF00290976

Broadbent, D., 1984. Mental Models. Q. J. Exp. Psychol. Sect. A 36, 673-681. https://doi.org/10.1080/14640748408402186

Brown, J.D., 2002. The Cronbach alpha reliability estimate The Cronbach alpha reliability estimate How should we interpret Cronbach alpha? JALT Test. Eval. SIG Newsl. 6, 1718.

Brown, R., Hewstone, M., 2005. An integrative theory of intergroup contact. Adv. Exp. Soc. Psychol. 37, 255-343. https://doi.org/10.1016/S0065-2601(05)37005-5

Brünken, R., Plass, J.L., Leutner, D., 2004. Assessment of cognitive load in multimedia learning with dual-task methodology: Auditory load and modality effects. Instr. Sci. 32, 115-132. https://doi.org/10.1023/b:truc.0000021812.96911.c5

Caporael, L.R., 1986. Anthropomorphism and mechanomorphism: Two faces of the human machine. Comput. Human Behav. 2, 215-234. https://doi.org/10.1016/07475632(86)90004-X

Carlston, D.E., Smith, E.R., 2007. Principles of mental representation. Soc. Psychol. Handb. basic Princ.

Carpinella, C.M., Wyman, A.B., Perez, M.A., Stroessner, S.J., 2017. The Robotic Social Attributes Scale (RoSAS): Development and Validation, in: ACM/IEEE International 
Conference on Human-Robot Interaction. pp. 254-262. https://doi.org/10.1145/2909824.3020208

Chaminade, T., 2017. An experimental approach to study the physiology of natural social interactions. Interact. Stud. Soc. Behav. Commun. Biol. Artif. Syst. Stud. / Soc. Behav. Commun. Biol. Artif. Syst. Stud. 18, 254-275. https://doi.org/10.1075/is.18.2.06gry

Chaminade, T., Franklin, D.W., Oztop, E., Cheng, G., 2005. Motor interference between humans and humanoid robots: Effect of biological and artificial motion, in: Proceedings of 2005 4th IEEE International Conference on Development and Learning. pp. 96-101. https://doi.org/10.1109/DEVLRN.2005.1490951

Chaminade, T., Rosset, D., Da Fonseca, D., Nazarian, B., Lutcher, E., Cheng, G., Deruelle, C., 2012. How do we think machines think? An fMRI study of alleged competition with an artificial intelligence. Front. Hum. Neurosci. 6. https://doi.org/10.3389/fnhum.2012.00103

Chaminade, T., Zecca, M., Blakemore, S.J., Takanishi, A., Frith, C.D., Micera, S., Dario, P., Rizzolatti, G., Gallese, V., Umiltà, M.A., 2010. Brain response to a humanoid robot in areas implicated in the perception of human emotional gestures. PLoS One 5. https://doi.org/10.1371/journal.pone.0011577

Coeckelbergh, M., 2012. Growing moral relations: Critique of moral status ascription, Growing Moral Relations: Critique of Moral Status Ascription. https://doi.org/10.1057/9781137025968

Cortina, J.M., 1993. What Is Coefficient Alpha? An Examination of Theory and Applications. J. Appl. Psychol. 78, 98-104. https://doi.org/10.1037/0021-9010.78.1.98

Cronbach, L.J., 1951. Coefficient alpha and the internal structure of tests. Psychometrika 16, 297-334. https://doi.org/10.1007/BF02310555 
Cunningham, W.A., Zelazo, P.D., 2007. Attitudes and evaluations: a social cognitive neuroscience perspective. Trends Cogn. Sci. 11, 97-104. https://doi.org/10.1016/j.tics.2006.12.005

Darling, K., 2017. "Who's johnny?" Anthropomorphic framing in human-robot: Interaction, integration, and policy, Robot Ethics 2.0: From Autonomous Cars to Artificial Intelligence. https://doi.org/10.1093/oso/9780190652951.003.0012

Darlow, A.L., Sloman, S.A., 2010. Two systems of reasoning: Architecture and relation to emotion. Wiley Interdiscip. Rev. Cogn. Sci. https://doi.org/10.1002/wcs.34

De Houwer, J., Teige-Mocigemba, S., Spruyt, A., Moors, A., 2009. Implicit Measures: A Normative Analysis and Review. Psychol. Bull. 135, 347-368. https://doi.org/10.1037/a0014211

de Lange, F.P., Spronk, M., Willems, R.M., Toni, I., Bekkering, H., 2008. Complementary Systems for Understanding Action Intentions. Curr. Biol. 18, 454-457. https://doi.org/10.1016/j.cub.2008.02.057

Dennett, D., 2009. Intentional Systems Theory, in: The Oxford Handbook of Philosophy of Mind. pp. 87-106. https://doi.org/10.1093/oxfordhb/9780199262618.003.0020

Dennett, D.C., 1971. Intentional Systems. J. Philos. 68, 87-106. https://doi.org/10.2307/2025382

DiSalvo, C.F., Gemperle, F., Forlizzi, J., Kiesler, S., 2002. All robots are not created equal: The design and perception of humanoid robot heads, in: Proceedings of the Conference on Designing Interactive Systems: Processes, Practices, Methods, and Techniques, DIS. pp. 321-326. https://doi.org/10.1145/778712.778756 
Duffy, B.R., 2003. Anthropomorphism and the social robot, in: Robotics and Autonomous Systems. pp. 177-190. https://doi.org/10.1016/S0921-8890(02)00374-3

Dumontheil, I., Wolf, L.K., Blakemore, S.J., 2016. Audience effects on the neural correlates of relational reasoning in adolescence. Neuropsychologia 87, 85-95. https://doi.org/10.1016/j.neuropsychologia.2016.05.001

Eddy, T.J., Gallup, G.G., Povinelli, D.J., 1993. Attribution of Cognitive States to Animals: Anthropomorphism in Comparative Perspective. J. Soc. Issues 49, 87-101. https://doi.org/10.1111/j.1540-4560.1993.tb00910.x

Eisenberger, N.I., Cole, S.W., 2012. Social neuroscience and health: Neurophysiological mechanisms linking social ties with physical health. Nat. Neurosci. https://doi.org/10.1038/nn.3086

Engle, R.W., 2002. Working memory capacity as executive attention. Curr. Dir. Psychol. Sci. 11, 19-23. https://doi.org/10.1111/1467-8721.00160

Engström, J., Markkula, G., Victor, T., Merat, N., 2017. Effects of Cognitive Load on Driving Performance: The Cognitive Control Hypothesis. Hum. Factors 59, 734-764. https://doi.org/10.1177/0018720817690639

Epley, N., Waytz, A., Akalis, S., Cacioppo, J.T., 2008. When we need a human: Motivational determinants of anthropomorphism. Soc. Cogn. 26, 143-155. https://doi.org/10.1521/soco.2008.26.2.143

Epley, N., Waytz, A., Cacioppo, J.T., 2007. On Seeing Human: A Three-Factor Theory of Anthropomorphism. Psychol. Rev. 114, 864-886. https://doi.org/10.1037/0033295X.114.4.864 
Evans, J.S.B.T., 2008. Dual-processing accounts of reasoning, judgment, and social cognition.

$\begin{array}{llll}\text { Annu. } & \text { Rev. } & \text { Psychol. } & \text { 59, }\end{array}$ https://doi.org/10.1146/annurev.psych.59.103006.093629

Evans, J.S.B.T., Stanovich, K.E., 2013. Dual-Process Theories of Higher Cognition: Advancing the Debate. Perspect. Psychol. Sci. 8, 223-241. https://doi.org/10.1177/1745691612460685

Eyssel, F., Kuchenbrandt, D., 2012. Social categorization of social robots: Anthropomorphism as a function of robot group membership. Br. J. Soc. Psychol. 51, 724-731. https://doi.org/10.1111/j.2044-8309.2011.02082.x

Faul, F., Erdfelder, E., Lang, A.G., Buchner, A., 2007. G*Power 3: A flexible statistical power analysis program for the social, behavioral, and biomedical sciences, in: Behavior Research Methods. pp. 175-191. https://doi.org/10.3758/BF03193146

Friese, M., Hofmann, W., Schmitt, M., 2008. When and why do implicit measures predict behaviour? Empirical evidence for the moderating role of opportunity, motivation, and process reliance. Eur. Rev. Soc. Psychol. 19, 285-338. https://doi.org/10.1080/10463280802556958

Fritz, M.S., Taylor, A.B., MacKinnon, D.P., 2012. Explanation of Two Anomalous Results in Statistical Mediation Analysis. Multivariate Behav. Res. 47, 61-87. https://doi.org/10.1080/00273171.2012.640596

Gazzola, V., Rizzolatti, G., Wicker, B., Keysers, C., 2007. The anthropomorphic brain: The mirror neuron system responds to human and robotic actions. Neuroimage 35, 1674-1684. https://doi.org/10.1016/j.neuroimage.2007.02.003

Geary, D.C., Widaman, K.F., Little, T.D., 1986. Cognitive addition and multiplication: 
Evidence for a single memory network. Mem. Cognit. 14, 478-487. https://doi.org/10.3758/BF03202519

Gilbert, D.T., Hixon, J.G., 1991. The Trouble of Thinking: Activation and Application of Stereotypic Beliefs. J. Pers. Soc. Psychol. 60, 509-517. https://doi.org/10.1037/00223514.60.4.509

Goodson, J.L., 2005. The vertebrate social behavior network: Evolutionary themes and variations. Horm. Behav. 48, 11-22. https://doi.org/10.1016/j.yhbeh.2005.02.003

Gunkel, D.J., 2019. The Machine Question, The Machine Question. https://doi.org/10.7551/mitpress/8975.001.0001

Gunkel, D.J., 2018. The other question: can and should robots have rights? Ethics Inf. Technol. 20, 87-99. https://doi.org/10.1007/s10676-017-9442-4

Hansen, R.J., Hinze, H., Holzer, H., 1976. Assay of phosphoenolpyruvate carboxykinase in crude yeast extracts, in: Analytical Biochemistry. pp. 576-584. https://doi.org/10.1016/0003-2697(76)90240-2

Haslam, N., 2006. Dehumanization: An integrative review. Personal. Soc. Psychol. Rev. 10, 252-264. https://doi.org/10.1207/s15327957pspr1003_4

Hayes, A.F., Rockwood, N.J., 2017. Regression-based statistical mediation and moderation analysis in clinical research: Observations, recommendations, and implementation. Behav. Res. Ther. 98, 39-57. https://doi.org/10.1016/j.brat.2016.11.001

Hayes, A.F., Scharkow, M., 2013. The Relative Trustworthiness of Inferential Tests of the Indirect Effect in Statistical Mediation Analysis: Does Method Really Matter? Psychol. Sci. 24, 1918-1927. https://doi.org/10.1177/0956797613480187 
Heider, F., Simmel, M., 1950. An Experimental Study of Apparent Behavior. Japanese J. Psychol. 20, 67-74. https://doi.org/10.4992/jjpsy.20.2_67

Heider, F., Simmel, M., 1944. An Experimental Study of Apparent Behavior. Japanese J. Psychol. 57, 243. https://doi.org/10.2307/1416950

Heil, J., Heil, J., 2019. The Intentional Stance, in: Philosophy of Mind. pp. 151-165. https://doi.org/10.4324/9780429506994-9

Heine, S.J., Proulx, T., Vohs, K.D., 2006. The meaning maintenance model: On the coherence of social motivations. Personal. Soc. Psychol. Rev. 10, 88-110. https://doi.org/10.1207/s15327957pspr1002_1

Hermans, D., Spruyt, A., De Houwer, J., Eelen, P., 2003. Affective priming with subliminally presented pictures. Can. J. Exp. Psychol. 57, 97-114. https://doi.org/10.1037/h0087416

Huang, L., Awh, E., 2018. Chunking in working memory via content-free labels. Sci. Rep. 8. https://doi.org/10.1038/s41598-017-18157-5

Humphrey, N., 1976. The social function of intellect, in: Growing Points in Ethology. Cambridge University Press, pp. 303-317.

Iacoboni, M., Lieberman, M.D., Knowlton, B.J., Molnar-Szakacs, I., Moritz, M., Throop, C.J., Fiske, A.P., 2004. Watching social interactions produces dorsomedial prefrontal and medial parietal BOLD fMRI signal increases compared to a resting baseline. Neuroimage 21, 1167-1173. https://doi.org/10.1016/j.neuroimage.2003.11.013

Iida, S., Nakao, T., Ohira, H., 2012. Prior cognitive activity implicitly modulates subsequent emotional responses to subliminally presented emotional stimuli. Cogn. Affect. Behav. Neurosci. 12, 337-345. https://doi.org/10.3758/s13415-012-0084-z 
Jack, A.I., Dawson, A.J., Begany, K.L., Leckie, R.L., Barry, K.P., Ciccia, A.H., Snyder, A.Z., 2013a. FMRI reveals reciprocal inhibition between social and physical cognitive domains. Neuroimage 66, 385-401. https://doi.org/10.1016/j.neuroimage.2012.10.061

Jack, A.I., Dawson, A.J., Norr, M.E., 2013b. Seeing human: Distinct and overlapping neural signatures associated with two forms of dehumanization. Neuroimage 79, 313-328. https://doi.org/10.1016/j.neuroimage.2013.04.109

Kelemen, D., Rosset, E., 2009. The Human Function Compunction: Teleological explanation in adults. Cognition 111, 138-143. https://doi.org/10.1016/j.cognition.2009.01.001

Keysers, C., Gazzola, V., 2007. Integrating simulation and theory of mind: from self to social cognition. Trends Cogn. Sci. https://doi.org/10.1016/j.tics.2007.02.002

Kuchenbrandt, D., Eyssel, F., Bobinger, S., Neufeld, M., 2013. When a Robot's Group Membership Matters: Anthropomorphization of Robots as a Function of Social Categorization. Int. J. Soc. Robot. 5, 409-417. https://doi.org/10.1007/s12369-013-01978

Kurdi, B., Seitchik, A.E., Axt, J.R., Carroll, T.J., Karapetyan, A., Kaushik, N., Tomezsko, D., Greenwald, A.G., Banaji, M.R., 2019. Relationship between the implicit association test and intergroup behavior: A meta-analysis. Am. Psychol. 74, 569-586. https://doi.org/10.1037/amp0000364

Lamberts, J., Van Den Broek, P.L.C., Bener, L., Van Egmond, J., Dirksen, R., Coenen, A.M.L., 2000. Correlation dimension of the human electroencephalogram corresponds with cognitive load. Neuropsychobiology 41, 149-153. https://doi.org/10.1159/000026647

Langer, E.J., 1992. Matters of mind: Mindfulness/mindlessness in perspective. Conscious. Cogn. 1, 289-305. https://doi.org/10.1016/1053-8100(92)90066-J 
Lavie, N., 2010. Attention, distraction, and cognitive control under load. Curr. Dir. Psychol. Sci. 19, 143-148. https://doi.org/10.1177/0963721410370295

Lee, S.L., Lau, I.Y.M., Kiesler, S., Chiu, C.Y., 2005. Human mental models of humanoid robots, in: Proceedings - IEEE International Conference on Robotics and Automation. pp. 2767-2772. https://doi.org/10.1109/ROBOT.2005.1570532

Leppink, J., van Gog, T., Paas, F., Sweller, J., 2015. Cognitive load theory: Researching and planning teaching to maximise learning. Res. Med. Educ. 207-218. https://doi.org/10.1002/9781118838983.ch18

Levy, D., 2009. The ethical treatment of artificially conscious robots. Int. J. Soc. Robot. 1, 209216. https://doi.org/10.1007/s12369-009-0022-6

Lin, S., Keysar, B., Epley, N., 2010. Reflexively mindblind: Using theory of mind to interpret behavior requires effortful attention. J. Exp. Soc. Psychol. 46, 551-556. https://doi.org/10.1016/j.jesp.2009.12.019

MacKinnon, D.P., Lockwood, C.M., Williams, J., 2004. Confidence limits for the indirect effect: Distribution of the product and resampling methods. Multivariate Behav. Res. 39, 99-128. https://doi.org/10.1207/s15327906mbr3901_4

MacLeod, C.M., 1992. The Stroop task: The "gold standard" of attentional measures. J. Exp. Psychol. Gen. 121, 12-14. https://doi.org/10.1037/0096-3445.121.1.12

Mars, R.B., Neubert, F.X., Noonan, M.A.P., Sallet, J., Toni, I., Rushworth, M.F.S., 2012. On the relationship between the "default mode network" and the "social brain." Front. Hum. Neurosci. 6, 1-9. https://doi.org/10.3389/fnhum.2012.00189

Marsh, L.E., Mullett, T.L., Ropar, D., Hamilton, A.F. d. C., 2014. Responses to irrational 
actions in action observation and mentalising networks of the human brain. Neuroimage 103, 81-90. https://doi.org/10.1016/j.neuroimage.2014.09.020

Meisner, B.A., 2012. A meta-analysis of positive and negative age stereotype priming effects on behavior among older adults. Journals Gerontol. - Ser. B Psychol. Sci. Soc. Sci. 67 B, 13-17. https://doi.org/10.1093/geronb/gbr062

Meyer, M.L., Spunt, R.P., Berkman, E.T., Taylor, S.E., Lieberman, M.D., 2012. Evidence for social working memory from a parametric functional MRI study. Proc. Natl. Acad. Sci. U. S. A. 109, 1883-1888. https://doi.org/10.1073/pnas.1121077109

Moors, A., De Houwer, J., 2006. Automaticity: A theoretical and conceptual analysis. Psychol. Bull. https://doi.org/10.1037/0033-2909.132.2.297

Moscovitch, M., 1994. Cognitive Resources and Dual-Task Interference Effects at Retrieval in Normal People: The Role of the Frontal Lobes and Medial Temporal Cortex. Neuropsychology 8, 524-534. https://doi.org/10.1037/0894-4105.8.4.524

Nass, C., Moon, Y., 2000. Machines and mindlessness: Social responses to computers. J. Soc. Issues 56, 81-103. https://doi.org/10.1111/0022-4537.00153

Neely, E.L., 2014. Machines and the moral community. Philos. Technol. 27, 97-111. https://doi.org/10.1007/s13347-013-0114-y

Nyangoma, E.N., Olson, C.K., Painter, J.A., Posey, D.L., Stauffer, W.M., Naughton, M., Zhou, W., Kamb, M., Benoit, S.R., 2017. Syphilis Among U.S.-Bound Refugees, 2009-2013, in: Journal of Immigrant and Minority Health. pp. 835-842. https://doi.org/10.1007/s10903016-0397-z

O’Rourke, J., 1993. Consciousness explained, Artificial Intelligence. Penguin uk. 
https://doi.org/10.1016/0004-3702(93)90006-W

Paas, F.G., Van Merriënboer, J.J., Adam, J.J., 1994. Measurement of cognitive load in instructional research. Percept. Mot. Skills 79, 419-430. https://doi.org/10.2466/pms.1994.79.1.419

Preacher, K.J., Hayes, A.F., 2004. SPSS and SAS procedures for estimating indirect effects in simple mediation models. Behav. Res. Methods, Instruments, Comput. 36, 717-731. https://doi.org/10.3758/BF03206553

Rauchbauer, B., Nazarian, B., Bourhis, M., Ochs, M., Prévot, L., Chaminade, T., 2019. Brain activity during reciprocal social interaction investigated using conversational robots as control condition. Philos. Trans. R. Soc. B Biol. Sci. 374. https://doi.org/10.1098/rstb.2018.0033

Ridderinkhof, K.R., Ullsperger, M., Crone, E.A., Nieuwenhuis, S., 2004a. The role of the medial frontal cortex in cognitive control. Science (80-. ). https://doi.org/10.1126/science.1100301

Ridderinkhof, K.R., Van Den Wildenberg, W.P.M., Segalowitz, S.J., Carter, C.S., 2004b. Neurocognitive mechanisms of cognitive control: The role of prefrontal cortex in action selection, response inhibition, performance monitoring, and reward-based learning. Brain Cogn. https://doi.org/10.1016/j.bandc.2004.09.016

Riether, N., Hegel, F., Wrede, B., Horstmann, G., 2012. Social facilitation with social robots?, in: HRI'12 - Proceedings of the 7th Annual ACM/IEEE International Conference on Human-Robot Interaction. pp. 41-47. https://doi.org/10.1145/2157689.2157697

Rosset, E., 2008. It's no accident: Our bias for intentional explanations. Cognition 108, 771780. https://doi.org/10.1016/j.cognition.2008.07.001 
Salem, M., Eyssel, F., Rohlfing, K., Kopp, S., Joublin, F., 2013. To Err is Human(-like): Effects of Robot Gesture on Perceived Anthropomorphism and Likability. Int. J. Soc. Robot. 5, 313-323. https://doi.org/10.1007/s12369-013-0196-9

Saris, I.M.J., Penninx, B.W.J.H., Dinga, R., van Tol, M.J., Veltman, D.J., van der Wee, N.J.A., Aghajani, M., 2020. Default Mode Network Connectivity and Social Dysfunction in Major Depressive Disorder. Sci. Rep. 10, 1-11. https://doi.org/10.1038/s41598-019-57033-2

Schilbach, L., Eickhoff, S.B., Rotarska-Jagiela, A., Fink, G.R., Vogeley, K., 2008. Minds at rest? Social cognition as the default mode of cognizing and its putative relationship to the "default system" of the brain. Conscious. Cogn. 17, 457-467. https://doi.org/10.1016/j.concog.2008.03.013

Sharma, D., Booth, R., Brown, R., Huguet, P., 2010. Exploring the temporal dynamics of social facilitation in the stroop task. Psychon. Bull. Rev. 17, 52-58. https://doi.org/10.3758/PBR.17.1.52

Sherman, J.W., Frost, L.A., 2000. On the encoding of stereotype-relevant information under cognitive load. Personal. Soc. Psychol. Bull. 26, 26-34. https://doi.org/10.1177/0146167200261003

Sherman, J.W., Lee, A.Y., Bessenoff, G.R., Frost, L.A., 1998. Stereotype Efficiency Reconsidered: Encoding Flexibility under Cognitive Load. J. Pers. Soc. Psychol. 75, 589606. https://doi.org/10.1037/0022-3514.75.3.589

Shrout, P.E., Bolger, N., 2002. Mediation in experimental and nonexperimental studies: New procedures and recommendations. Psychol. Methods 7, 422-445. https://doi.org/10.1037/1082-989X.7.4.422

Smith, E.R., DeCoster, J., 2000. Dual-process models in social and cognitive psychology: 
Conceptual integration and links to underlying memory systems. Personal. Soc. Psychol. Rev. 4, 108-131. https://doi.org/10.1207/S15327957PSPR0402_01

Snyder, R.G., 1961. Vibrational spectra of crystalline n-paraffins. II. Intermolecular effects. J. Mol. Spectrosc. https://doi.org/10.1016/0022-2852(61)90347-2

Spatola, N., Belletier, C., Chausse, P., Augustinova, M., Normand, A., Barra, V., Ferrand, L., Huguet, P., 2019. Improved Cognitive Control in Presence of Anthropomorphized Robots. Int. J. Soc. Robot. 11, 463-476. https://doi.org/10.1007/s12369-018-00511-w

Spatola, N., Belletier, C., Normand, A., Chausse, P., Monceau, S., Augustinova, M., Barra, V., Huguet, P., Ferrand, L., 2018. Not as bad as it seems: When the presence of a threatening humanoid robot improves human performance. Sci. Robot. 3, eaat5843. https://doi.org/10.1126/scirobotics.aat5843

Spatola, N., Hmamouche, Y., Chaminade, T., n.d. Social brain activity evolution during reciprocal Human-Robot and Human-Human social interaction.

Spatola, N., Kühnlenz, B., Cheng, G., 2020. Perception and evaluation in human-robot interaction: The Human-Robot Interaction Evaluation Scale (HRIES) - a multicomponent approach of anthropomorphism. Int. J. Soc. Robot. https://doi.org/10.1007/s12369-02000667-4

Spatola, N., Urbanska, K., 2019. God-like robots: the semantic overlap between representation of divine and artificial entities. AI Soc. 35, 329-341. https://doi.org/10.1007/s00146-01900902-1

Spatola, N., Wudarczyk, O., 2020. Implicit Attitudes Towards Robots Predict Explicit Attitudes, Semantic Distance Between Robots and Humans, Anthropomorphism, and Prosocial Behavior: From Attitudes to Human-Robot Interaction. Int. J. Soc. Robot. 1-11. 
https://doi.org/10.1007/s12369-020-00701-5

Spatola, N., Wudarczyk, O.A., 2021. Ascribing emotions to robots: Explicit and implicit attribution of emotions and perceived robot anthropomorphism. Comput. Human Behav. https://doi.org/10.1016/j.chb.2021.106934

Sperduti, M., Guionnet, S., Fossati, P., Nadel, J., 2014. Mirror Neuron System and Mentalizing System connect during online social interaction. Cogn. Process. 15, 307-316. https://doi.org/10.1007/s10339-014-0600-x

Spunt, R.P., Lieberman, M.D., 2013. The Busy Social Brain: Evidence for Automaticity and Control in the Neural Systems Supporting Social Cognition and Action Understanding. Psychol. Sci. 24, 80-86. https://doi.org/10.1177/0956797612450884

Spunt, R.P., Meyer, M.L., Lieberman, M.D., 2015. The default mode of human brain function primes the intentional stance. J. Cogn. Neurosci. 27, 1116-1124. https://doi.org/10.1162/jocn_a_00785

Steindl, C., Jonas, E., Sittenthaler, S., Traut-Mattausch, E., Greenberg, J., 2015. Understanding psychological reactance: New developments and findings. Zeitschrift fur Psychol. / J. Psychol. https://doi.org/10.1027/2151-2604/a000222

Stroop, J.R., 1935. Studies of interference in serial verbal reactions. J. Exp. Psychol. 18, 643662. https://doi.org/10.1037/h0054651

Sundar, S.S., Waddell, T.F., Jung, E.H., 2016. The Hollywood robot syndrome: Media effects on older adults' attitudes toward robots and adoption intentions, in: ACM/IEEE International Conference on Human-Robot Interaction. pp. 343-350. https://doi.org/10.1109/HRI.2016.7451771 
Sweller, J., 1994. Cognitive load theory, learning difficulty, and instructional design. Learn. Instr. 4, 295-312. https://doi.org/10.1016/0959-4752(94)90003-5

Syrdal, D.S., Dautenhahn, K., Koay, K.L., Walters, M.L., 2009. The Negative Attitudes Towards Robots Scale and reactions to robot behaviour in a live Human-Robot Interaction study, in: Adaptive and Emergent Behaviour and Complex Systems - Proceedings of the 23rd Convention of the Society for the Study of Artificial Intelligence and Simulation of Behaviour, AISB 2009. pp. 109-115. https://doi.org/10.1.1.159.9791

Tavares, P., Lawrence, A.D., Barnard, P.J., 2008. Paying attention to social meaning: An fMRI study. Cereb. Cortex 18, 1876-1885. https://doi.org/10.1093/cercor/bhm212

Urquiza-Haas, E.G., Kotrschal, K., 2015. The mind behind anthropomorphic thinking: Attribution of mental states to other species. Anim. Behav. 109, 167-176. https://doi.org/10.1016/j.anbehav.2015.08.011

Valtorta, N.K., Kanaan, M., Gilbody, S., Hanratty, B., 2018. Loneliness, social isolation and risk of cardiovascular disease in the English Longitudinal Study of Ageing. Eur. J. Prev. Cardiol. 25, 1387-1396. https://doi.org/10.1177/2047487318792696

Van Overwalle, F., 2009. Social cognition and the brain: A meta-analysis. Hum. Brain Mapp. https://doi.org/10.1002/hbm.20547

Van Overwalle, F., Baetens, K., 2009. Understanding others' actions and goals by mirror and mentalizing $\quad$ systems: $\quad$ A meta-analysis. https://doi.org/10.1016/j.neuroimage.2009.06.009

Waytz, A., Cacioppo, J., Epley, N., 2010. Who sees human? The stability and importance of individual differences in anthropomorphism. Perspect. Psychol. Sci. 5, 219-232. https://doi.org/10.1177/1745691610369336 
Wegner, D.M., Bargh, J. a, 1998. Control and automaticity in social life, in: Gilbert, D., Fiske, S.T., Lindzey, G. (Eds.), Handbook of Social Psychology. McGraw-Hill, New York, pp. $446-496$.

Wegner, D.M., Schneider, D.J., 2003. The White Bear Story. Psychol. Inq. 14, 326-329. https://doi.org/10.1207/s15327965pli1403\&4_24

Wehling, T.O., Lichtenstein, A.I., Katsnelson, M.I., 2008. First-principles studies of water adsorption on graphene: The role of the substrate. Appl. Phys. Lett. 93, 196-210. https://doi.org/10.1063/1.3033202

Wheatley, T., Milleville, S.C., Martin, A., 2007. Understanding animate agents: Distinct roles for the social network and mirror system: Research report. Psychol. Sci. 18, 469-474. https://doi.org/10.1111/j.1467-9280.2007.01923.x

Wynne, C.D.L., 2006. What are Animals? Why Anthropomorphism is Still Not a Scientific Approach to Behavior. Comp. Cogn. Behav. Rev. 2. https://doi.org/10.3819/ccbr.2008.20008

Yzerbyt, V., Muller, D., Batailler, C., Judd, C.M., 2018. New recommendations for testing indirect effects in mediational models: The need to report and test component paths. $\mathrm{J}$. Pers. Soc. Psychol. 115, 929-943. https://doi.org/10.1037/pspa0000132

Złotowski, J., Proudfoot, D., Yogeeswaran, K., Bartneck, C., 2015. Anthropomorphism: Opportunities and Challenges in Human-Robot Interaction. Int. J. Soc. Robot. 7, 347-360. https://doi.org/10.1007/s12369-014-0267-6 